\title{
Logarithmic Vanishing Theorems and
} Arakelov-Parshin Boundedness for Singular Varieties

\author{
SÁNDOR J. KOVÁCS* \\ Department of Mathematics, University of Washington, Box 354350, Seattle, WA 98103, \\ U.S.A.E-mail:kovacs@math.washington.edu
}

(Received: 11 April 2000; accepted in final form: 7 March 2001)

\begin{abstract}
This article can be divided into two loosely connected parts. The first part is devoted to proving a singular version of the logarithmic Kodaira-Akizuki-Nakano vanishing theorem of Esnault and Viehweg in the style of Navarro-Aznar et al. This in turn is used to prove other vanishing theorems. In the second part, these vanishing theorems are used to prove an Arakelov-Parshin type boundedness result for families of canonically polarized varieties with rational Gorenstein singularities.
\end{abstract}

Mathematics Subject Classification (2000). 14J10.

Key words. families, general type, vanishing theorems, rational singularities.

Vanishing theorems have played a central role in algebraic geometry for the last couple of decades, especially in classification theory. Kollár [22] gives an introduction to the basic use of vanishing theorems as well as a survey of the results and applications available at the time. For more recent results, one should consult $[8,27,30,37]$. Because of the availability of such surveys, here we will only recall statements that are important to this article.

The classical Kodaira vanishing theorem has been generalized in many ways. A very important generalization is the Kodaira-Akizuki-Nakano vanishing theorem and even that has been further generalized, most notably in two different directions.

Esnault and Viehweg extended the theorem to sheaves of logarithmic differential forms:

THEOREM $0.1([11,6.4])$. Let $X$ be a smooth complex projective variety and $\mathcal{L}$ an ample line bundle on $X$. Further, let $D$ be a normal crossing divisor on $X$. Then

$$
H^{q}\left(X, \Omega_{X}^{p}(\log D) \otimes \mathcal{L}\right)=0 \quad \text { for } p+q>n .
$$

^Supported in part by an AMS Centennial Fellowship and by NSF Grants DMS-0196072 and DMS-0092165. 
Another type of generalization was proved by Navarro-Aznar and co-workers. They gave a generalization for singular varieties. To state their theorem, one requires a short introduction: For any scheme, $X$, of finite type over $\mathbb{C}$, there exists a filtered complex in the derived category of $\mathcal{O}_{X}$-modules, called Du Bois's complex, $\underline{\Omega}_{X}$, that resembles the De Rham complex of a smooth variety in many respect (1.2). Using the notation, $\underline{\Omega}_{X}^{p}:=G r_{\text {filt }}^{p} \underline{\Omega}_{X}[p]$ :

THEOREM $0.2([15,32])$. Let $\mathcal{L}$ be an ample line bundle on $X$. Then

$$
\mathbb{H}^{q}\left(X, \underline{\Omega}_{X}^{p} \otimes \mathcal{L}\right) \quad \text { for } p+q>\operatorname{dim} X .
$$

Of course, Du Bois's complex agrees with the De Rham complex for smooth varieties, so this theorem reduces to the Kodaira-Akizuki-Nakano theorem in the smooth case.

One of the main goals of the present article is to prove a common generalization of Theorems 0.1 and 0.2 . For simplicity, only a weaker statement is given here. A somewhat stronger version is given in (4.1).

To state the theorem, one needs a generalization of the logarithmic De Rham complex to the singular case. Fortunately, Du Bois's complex has a logarithmic version and again it resembles the smooth case very much (1.2).

MAIN THEOREM 0.3. Let $X$ be a complex projective variety and $\mathcal{L}$ an ample line bundle on $X$. Further, let $D$ be a normal crossing divisor on $X$. Then

$$
\mathbb{H}^{q}\left(X, \underline{\Omega}_{X}^{p}(\log D) \otimes \mathcal{L}\right)=0 \quad \text { for } p+q>n .
$$

One could ask why we need such a generalization. I believe it is an interesting result on its own. This seems to be supported by the enthusiasm that greeted Theorem 0.2 (cf. [38]). On the other hand, it could be viewed as a 'poor man's version' of the logarithmic Kodaira-Akizuki-Nakano vanishing theorem for semi-ample and big line bundles on smooth varieties. Considering that the obvious generalization fails, this might be the best one can hope for. As an easy corollary, we also obtain a relative version of this vanishing theorem.

Nevertheless, my original motivation was an actual application. This theorem is the cornerstone of the proof of an Arakelov-Parshin-type boundedness result. That result is presented as an application of the Main Theorem, although it would merit to be called a 'Main Theorem' itself.

The first interesting consequence of the Main Theorem is a vanishing theorem for smooth varieties, (6.4). Note that in order to prove it one has to go through the singular version. Theorem 6.4 is a generalization of $[25,1.1]$ and similar in nature to $[5,2.2]$.

Next, let us take a brief tour of some related problems, and let us start by recalling that a family of projective curves is called isotrivial if all but finitely many members of the family are isomorphic to a fixed curve. 
0.4. Fix $C$, a smooth projective curve of genus $g$ over an algebraically closed field of characteristic $0, \Delta \subset C$ a finite subset and $q>1$ positive integer. Let $\delta=\# \Delta$.

Shafarevich conjectured at the 1962 ICM in Stockholm that the set, $\mathbb{S}$, of nonisotrivial families of smooth projective curves of genus $q$ over $C \backslash \Delta$ is finite. and that if $2 q-2+\delta \leqslant 0$ then no such families exist.

This was confirmed by Parshin [34] for the case $\Delta=\emptyset$ and by Arakelov [3] in general. Their method was to divide the problem into the following parts:

(1) 'Boundedness': There are only finitely many deformation types of families in $\subseteq$.

(2) 'Rigidity': There are no nontrivial deformations within $\subseteq$.

(3) 'Hyperbolicity': If $2 g-2+\delta \leqslant 0$, then $\subseteq=\emptyset$.

The basic question now is whether (an appropriate variant of) Shafarevich's conjecture holds in higher dimensions. It seems natural to try to work with these parts individually as they may generalize in different ways.

0.5. It is actually more convenient to work with a compactification of the family, understanding that later we are free to alter it over $\Delta$. Let $\bar{\varsigma}$ be the set of nonisotrivial families of projective curves of genus $q$ over $C$ that are smooth over $C \backslash \Delta$ and have a smooth total space.

The first important observation is that 'boundedness' follows from the existence of moduli spaces of curves and from

$\left(1^{\prime}\right)$ 'Weak boundedness': There exist a function $d(g, \delta, q, m)$, and a natural number $m_{0}$, such that for any family $f: X \rightarrow C$ in $\widetilde{\mathfrak{S}}, \operatorname{deg}\left(f_{*} \omega_{X / C}^{m}\right) \leqslant d(g, \delta, q, m)$ for all $m \geqslant m_{0}$.

0.6. Considering families over a compact base curve leads to a slightly different view on the hyperbolicity problem. One could ask what can be said about the singular fibers of the family. On the simplest level, how many are there? In fact Szpiro did ask this: Is there a lower bound on the number of singular fibers if $C \simeq \mathbb{P}^{1}$ ?

Beauville [4] gave the following answer: there are always at least 3 singular fibers and there are families with exactly 3 . In fact, Beauville's proof also shows that there is at least 1 singular fiber if the base curve is elliptic. In short $2 g-2+\delta>0$, i.e., 'hyperbolicity' holds.

Note that Kodaira surfaces show that there are families over high genus curves without any singular fibers.

More recently, Catanese and Schneider [6] asked if the same is true with higherdimensional fibers, and the conjecture of Shokurov [36] translates to the same: Is it true that for a familily of varieties of general type, $\delta \geqslant 3$ if $g=0$ and $\delta \geqslant 1$ if $g=1$, or, equivalently, Is $2 g-2+\delta>0$ ? This is again simply asking if 'hyperbolicity' also holds in higher dimensions.

It is interesting to note the wide range of applications this question relates to. Catanese and Schneider [6] wanted to use this to obtain good estimates for the size 
of the automorphism group of a variety of general type, while Shokurov [36] needed it for proving quasi-projectivity of certain moduli spaces.

0.7. The following is a select list of results related to these questions.

Faltings [12] studied the Shafarevich problem for families of Abelian varieties and proved that boundedness holds, while rigidity fails in general.

Migliorini [31] showed that for families of minimal surfaces $\delta \geqslant 1$ if $g \leqslant 1$.

Kovács [26] showed the same for families of minimal varieties of arbitrary dimension, [27] settled the question for families of minimal surfaces, and [28] for families of canonically polarized varieties: In all cases $2 g-2+\delta>0$.

Oguiso and Viehweg [33] proved the same for families of elliptic surfaces. Their work completes the case of families of minimal varieties of nonnegative Kodaira dimension.

Bedulev and Viehweg [5] proved that boundedness holds for families of surfaces of general type and that weak boundedness (and in some cases boundedness) holds for families of canonically polarized varieties. As a byproduct of their proof they also obtained that $2 g-2+\delta>0$ in these cases.

In this article we obtain results regarding boundedness and hyperbolicity. In fact a simple observation yields that these questions are strongly related.

THEOREM 0.8. 'Weak boundedness' implies 'hyperbolicity'.

A more precise and somewhat more general formulation is the following:

THEOREM 0.9. Let ₹ be a collection of smooth varieties of general type, $C$ a smooth projective curve and $\Delta \subset C$ a finite subset of $C$. Let

$$
\begin{aligned}
& \mathcal{F} \text { am }(C, \Delta, \mathfrak{F})= \\
&\left\{f: X \rightarrow C \mid X \text { is smooth, } f \text { is flat and } f^{-1}(t) \in \mathfrak{F}, \text { for all } t \in C \backslash \Delta\right\} .
\end{aligned}
$$

Assume that there exist $M, m \in \mathbb{N}$ such that for all $(f: X \rightarrow C) \in \mathcal{F}$ am $(C, \Delta, \mathfrak{F})$,

$$
\operatorname{deg}\left(f_{*} \omega_{X / C}^{m}\right) \leqslant M
$$

and that $\mathcal{F} a m(C, \Delta, \mathfrak{F})$ contains nonisotrivial families. Then $2 g-2+\delta>0$.

Proof. Assume the contrary, i.e., either $g=0$ and $\delta \leqslant 2$ or $g=1$ and $\delta=0$. This allows us to assume that $f: X \rightarrow C$ is semi-stable and nonisotrivial. Also, in both cases there exists a finite endomorphism, $\tau$ : $C \rightarrow C$, of degree $>1$ such that $\tau$ is smooth over $C \backslash \Delta$ and completely ramified over $\Delta$.

Let $\pi: \tilde{X}_{\tau} \rightarrow X_{\tau}$ be a resolution of singularities that is an isomorphism over $C \backslash \Delta$, and $\tilde{f}_{\tau}=f_{\tau} \circ \pi$. Then $\left(\tilde{f}_{\tau}: \tilde{X}_{\tau} \rightarrow C_{\tau}\right) \in \mathcal{F}$ am $(C, \Delta, \tilde{F})$. In particular $C_{\tau} \simeq C$ and $f_{\tau}$ is smooth over $C_{\tau} \backslash \Delta_{\tau} \simeq C \backslash \Delta$ with fibers in $\mathfrak{F}$.

Therefore, by assumption, $\operatorname{deg} \tau \cdot \operatorname{deg}\left(f_{*} \omega_{X / C}^{m}\right)=\operatorname{deg}\left(f_{\tau_{*}} \omega_{X_{\tau} / C_{\tau}}^{m}\right) \leqslant M$ as well. By iterating this process, $\operatorname{deg} \tau$ can grow arbitrary large and since $\operatorname{deg}\left(f_{*} \omega_{X / C}^{m}\right)>0$ by [22], $\operatorname{deg}\left(f_{\tau_{*}} \omega_{X_{\tau} / C_{\tau}}^{m}\right)$ cannot be bounded. 
The main result of the second part of the article is the following. It is in a non-explicit form. For more precise statements, see Lemma 7.8, Corollaries 7.10, 7.11 and Theorem 7.13.

THEOREM 0.10. Fix $C, \Delta \subset C$. Then weak boundedness holds for families of canonically polarized varieties with rational Gorenstein singularities over $C \backslash \Delta$ with fixed Hilbert polynomial admitting a simultaneous resolution of singularities. In particular $2 g-2+\delta>0$ for these families by Theorem 0.9 .

As a corollary, one obtains weak boundedness for nonbirationally isotrivial families of minimal varieties of general type.

A few days before the completion of this article I learnt that Viehweg and Zuo [42] proved that $2 g-2+\delta>0$ holds for nonbirationally isotrivial smooth families of minimal varieties. As a byproduct of their proof they also obtain weak boundedness for these families.

DEFINITIONS AND NOTATIONS. Throughout the article, the groundfield will always be $\mathbb{C}$, the field of complex numbers. A complex scheme (resp. complex variety) will mean a separated scheme (resp. variety) of finite type over $\mathbb{C}$.

A divisor $D$ is called $\mathbb{Q}$-Cartier if $m D$ is Cartier for some $m>0$. $D$ is called big if $X$ is proper and $|m D|$ gives a birational map for some $m>0$ and it is called nef if $D . C \geqslant 0$ for every proper curve $C \subset X$. In particular ample implies nef and big. If $A$ and $B$ are effective divisors, then $A \cup B$ will denote $\operatorname{supp}(A+B)$.

Let $U$ be an open subset of $X$. A line bundle $\mathcal{L}$ on $X$ is called semi-ample with respect to $U$ if some positive power of $\mathcal{L}$ is generated by global sections over $U$. It is called semi-ample if it is semi-ample with respect to $X$. Similarly $\mathcal{L}$ is called ample with respect to $U$ if the global sections of some positive power of $\mathcal{L}$ define a rational map, that is an embedding on $U$.

A locally free sheaf $\mathcal{E}$ on a scheme $X$ is called semi-positive (resp. ample) if for every smooth complete curve $C$ and every map $\gamma: C \rightarrow X$, any quotient bundle of $\gamma^{*} \mathcal{E}$ has nonnegative (resp. positive) degree.

Let $f: X \rightarrow S$ be a morphism of schemes. Then $X_{s}$ denotes the fibre of $f$ over the point $s \in S$ and $f_{s}$ denotes the restriction of $f$ to $X_{s}$. More generally, for a morphism $\sigma: Z \rightarrow S$, let $f_{Z}: X_{Z}=X \times_{S} Z \rightarrow Z$. If $f$ is composed with another morphism $g: S \rightarrow T$, then for a $t \in T, X_{t}$ denotes the fibre of $g \circ f$ over the point $t$, i.e., $X_{t}=X_{S_{t}} . f_{Z}$ and $X_{Z}$ may also be denoted by $f_{\sigma}$ and $X_{\sigma}$, respectively.

A singularity is called Gorenstein if its local ring is a Gorenstein ring. A variety is Gorenstein if it admits only Gorenstein singularities. Let $X$ be a normal variety and $f: Y \rightarrow X$ a resolution of singularities. $X$ is said to have rational singularities if $R^{i} f_{*} \mathcal{O}_{Y}=0$ for all $i>0$.

Let $X$ be a complex scheme of dimension $n . D_{\text {filt }}(X)$ denotes the derived category of filtered complexes of $\mathcal{O}_{X}$-modules with differentials of order $\leqslant 1$ and $D_{\text {filt,coh }}(X)$ the subcategory of $D_{\text {filt }}(X)$ of complexes $K$, such that for all $i$, the cohomology sheaves of $G r_{\text {filt }}^{i} K^{\prime}$ are coherent (cf. [7, 15]). $D(X)$ and $D_{\text {coh }}(X)$ denotes the derived categories 
with the same definition except that the complexes are assumed to have the trivial filtration. The superscripts,,$+- b$ carry the usual meaning (bounded below, bounded above, bounded). $C(X)$ is the category of complexes of $\mathcal{O}_{X}$-modules with differentials of order $\leqslant 1$ and for $u \in \operatorname{Mor}(C(X)), M(u) \in \operatorname{Ob}(C(X))$ denotes the mapping cone of $u$ (cf. [16]). Isomorphism in these categories is denoted by $\simeq_{\text {qis }}$. If $K^{\prime}$ is a complex in any of the above defined categories, then $h^{i}\left(K^{\prime}\right)$ denotes the $i$ th cohomolgy sheaf of $K$. In particular, every sheaf is naturally a complex with $h^{i}=0$ for $i \neq 0$.

The right derived functor of an additive functor $F$, if exists, is denoted by $R F$ and $R^{i} F$ stands for $h^{i} \circ R F$. In particular, $\mathbb{H}^{i}$ denotes $R^{i} \Gamma$ and $\mathbb{H}_{Z}^{i}$ denotes $R^{i} \Gamma_{Z}$ and $\mathcal{H}_{Z}^{i}$ denotes $R^{i} \mathcal{H}_{Z}$ where $\Gamma$ is the functor of global sections and $\Gamma_{Z}$ is the functor of global sections with support in the closed subset $Z$ and $\mathcal{H}_{Z}$ is the functor of local sections with support in the closed subset $Z$. Note that according to this terminology if $\phi: Y \rightarrow X$ is a morphism and $\mathcal{F}$ is a coherent sheaf on $Y$, then $R \phi_{*} \mathcal{F}$ is the complex whose cohomology sheaves give the usual higher direct images of $\mathcal{F}$. The derived functor of $\otimes$ is denoted by $\otimes^{L}$.

The dimension of the empty set is $-\infty$.

\section{De Rham-Du Bois Complexes}

In order to state our generalized version of the Kodaira-Akizuki-Nakano vanishing theorem, we need Du Bois's generalized De Rham complex.

The original construction of Du Bois's complex, $\underline{\Omega}_{X}(\log D)$, is based on simplicial resolutions. The reader interested in the details is referred to the original article [7]. Note also that a simplified construction was later obtained in [15] via the general theory of cubic resolutions. An easily accessible introduction can be found in [38].

The word 'hyperresolution' will refer to either simplicial or cubic resolution. Formally the construction of $\underline{\Omega}_{X}(\log D)$ is the same regardless which resolution is used and no specific aspects of either resolution will be used.

DEFINITION. Let $X$ be a complex scheme and $D$ a closed subscheme whose complement is dense in $X$. Then $(X ., D$. $) \rightarrow(X, D)$ is a good hyperresolution if $X . X$ is a hyperresolution, and if $U .=X . \times_{X}(X \backslash D)$ and $D .=X$. $\backslash U$. then $D_{i}$ is a divisor with normal crossings on $X_{i}$ for all $i$.

THEOREM $1.2[7,6.3,6.5]$. Let $X$ be a proper complex scheme of finite type and $D$ a closed subscheme whose complement is dense in $X$. Then there exists a unique $\Omega_{X}(\log D) \in \mathrm{Ob}\left(D_{\mathrm{filt}}(X)\right)$ with the following properties, using the notation:

$$
\underline{\Omega}_{X}^{p}(D):=G r_{\text {filt }}^{p} \underline{\Omega}_{X}(\log D)[p] .
$$

(1.2.1) Let $j: X \backslash D \rightarrow X$ be the inclusion map. Then

$$
\underline{\Omega}_{X}(\log D) \simeq_{\mathrm{qis}} R j_{*} \mathbb{C}_{X \backslash D} .
$$


(1.2.2) It is functorial, i.e., if $\phi: Y \rightarrow X$ is a morphism of proper complex schemes of finite type, then there exists a natural map $\phi^{*}$ of filtered complexes

$$
\phi^{*}: \underline{\Omega}_{X}(\log D) \rightarrow R \phi_{*} \underline{\Omega}_{Y}\left(\log \phi^{*} D\right) .
$$

Furthermore, $\underline{\Omega}_{X}(\log D) \in \mathrm{Ob}\left(D_{\mathrm{filt}, \mathrm{coh}}^{b}(X)\right)$ and if $\phi$ is proper, then $\phi^{*}$ is a morphism in $D_{\text {filt,coh }}^{b}(X)$.

(1.2.3) Let $U \subseteq X$ be an open subscheme of $X$. Then

$$
\left.\underline{\Omega}_{X}(\log D)\right|_{U} \simeq_{\text {qis }} \underline{\Omega}_{U}\left(\left.\log D\right|_{U}\right) .
$$

(1.2.4) There exists a spectral sequence degenerating at $E_{1}$ and abutting to the singular cohomology of $X \backslash D$ :

$$
E_{1}^{p q}=\mathbb{H}^{q}\left(X, \underline{\Omega}_{X}^{p}(\log D)\right) \Rightarrow H^{p+q}(X \backslash D, \mathbb{C}) .
$$

(1.2.5) If $\epsilon .:(X ., D.) \rightarrow(X, D)$ is a good hyperresolution, then

$$
\underline{\Omega}_{X}(\log D) \simeq_{\text {qis }} R \epsilon_{* *} \Omega_{X}(\log D \text {.). }
$$

In particular $h^{i}\left(\underline{\Omega}_{X}^{p}(\log D)\right)=0$ for $i<0$.

(1.2.6) There exists a natural map, $\mathcal{O}_{X} \rightarrow \underline{\Omega}_{X}^{0}(\log D)$, compatible with (1.2.2).

(1.2.7) If $X$ is smooth and $D$ is a normal crossing divisor, then

$$
\underline{\Omega}_{X}(\log D) \simeq_{\text {qis }} \Omega_{X}(\log D) .
$$

In particular

$$
\underline{\Omega}_{X}^{p}(\log D) \simeq_{\mathrm{qis}} \Omega_{X}^{p}(\log D) .
$$

(1.2.8) If $\phi: Y \rightarrow X$ is a resolution of singularities, then

$$
\underline{\Omega}_{X}^{\operatorname{dim} X}(\log D) \simeq_{\mathrm{qis}} R \phi_{*} \omega_{Y}\left(\phi^{*} D\right) .
$$

\section{A Short Exact Sequence}

The following notation and assumptions will be used throughout this and the next section.

2.1. Let $X$ be a projective variety and $D \subset X$ an effective divisor on $X$ and $\varepsilon .:(X, D.) \rightarrow(X, D)$ a good hyperresolution. Let $\mathcal{M}$ be a semi-ample line bundle on $X$. Assume that $\mathcal{M}$ is ample with respect to $X \backslash D$. Let $\mathcal{L}=\mathcal{M}^{N}$ for some $N \gg 0, \sigma \in H^{0}(X, \mathcal{L})$ a general section, and $L=(\sigma=0)$. Note that $\mathcal{L}$ is generated by global sections and the morphism given by its global sections is an embedding on $X \backslash D$. In particular $L$ is transversal to $\varepsilon .:(X ., D.) \rightarrow(X, D)$. Finally let $D^{L}=\left.{ }^{D}\right|_{L}, \mathcal{M}_{L}=\left.\mathcal{M}\right|_{L}$, and $\mathcal{L}_{L}=\left.\mathcal{L}\right|_{L}$ 
Now $\lambda .=\varepsilon .\left.\right|_{L}:\left(L ., D_{.}^{L}\right) \rightarrow\left(L, D^{L}\right)$ is a good hyperresolution, where $L .=X . \times_{X} L$. Furthermore, by (1.2.5),

$$
\begin{aligned}
\underline{\Omega}_{X}(\log D) & =R \varepsilon_{* *} \Omega_{X}(\log D .), \\
\underline{\Omega}_{L}\left(\log D^{L}\right) & =R \lambda_{* *} \Omega_{L}\left(\log D^{L}\right) .
\end{aligned}
$$

LEMMA 2.2 One has the following distinguished triangle:

$$
\underline{\Omega}_{L}^{p-1}\left(\log D^{L}\right) \longrightarrow \underline{\Omega}_{X}^{p}(\log D) \otimes{ }^{L} \mathcal{L}_{L} \longrightarrow \underline{\Omega}_{L}^{p}\left(\log D^{L}\right) \otimes \mathcal{L}_{L} \stackrel{+1}{\longrightarrow} .
$$

Proof. First assume that $X$ is smooth and $D$ is an effective normal crossing divisor. Then one has the following commutative diagram, $[11,2.3]$ :

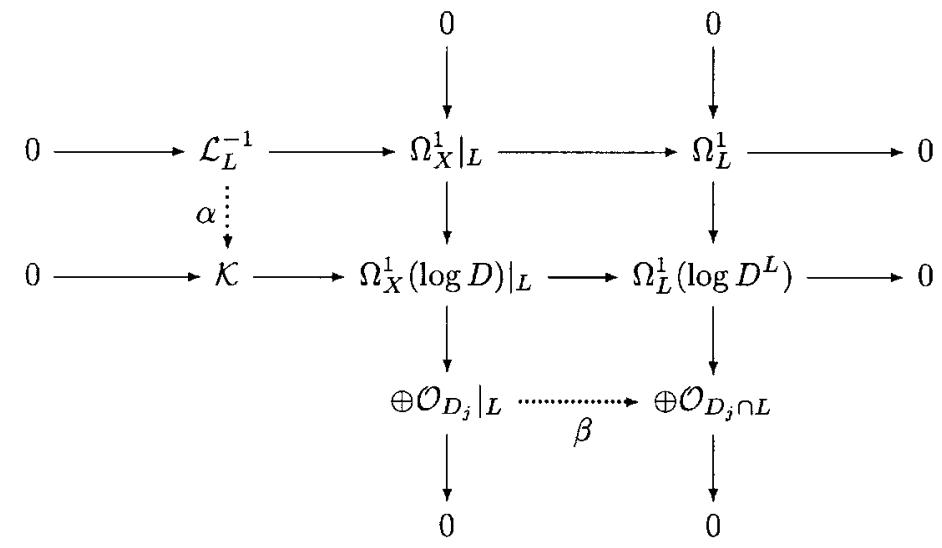

$L$ is transversal to $D$, so $\beta$ is an isomorphism, hence so is $\alpha$. Taking exterior powers one obtains that for all $p$ :

$$
\left.0 \longrightarrow \Omega_{L}^{p-1}\left(\log D^{L}\right) \otimes \mathcal{L}_{L}^{-1} \longrightarrow \Omega_{X}^{p}(\log D)\right|_{L} \longrightarrow \Omega_{L}^{p}\left(\log D^{L}\right) \longrightarrow 0 .
$$

Next consider the general case. Let $\varepsilon .:(X ., D.) \rightarrow(X, D)$ be a good hyperresolution. By (2.2.2) one has the following short exact sequence for all $i$ :

$$
0 \longrightarrow \Omega_{L_{i}}^{p-1}\left(\log D_{i}^{L_{i}}\right) \longrightarrow \Omega_{X_{i}}^{p}\left(\log D_{i}\right) \otimes \mathcal{L}_{L_{i}} \longrightarrow \Omega_{L_{i}}^{p}\left(\log D_{i}^{L_{i}}\right) \otimes \mathcal{L}_{L_{i}} \longrightarrow 0
$$

Since $L_{i}$ is the pull-back of $L$ for all $i$ these maps are compatible with $\lambda$, and then applying $R \lambda_{* *}$ gives the required distinguished triangle.

\section{Trace Map, Gysin Morphism, etc.}

The first subsection of this section is an adaptation of some parts of [17, II.2-3] to the logarithmic setting. 


\subsection{THE TRACE MAP}

3.1.1. In addition to the notation and assumptions of (2.1), $X$ and $L$ will be assumed to be smooth and $D$ an effective normal crossing divisor throughout this subsection. Consider the following short exact sequence,

$$
\left.0 \longrightarrow \mathcal{L}_{L}^{-1} \longrightarrow \Omega_{X}^{1}(\log D)\right|_{L} \longrightarrow \Omega_{L}^{1}\left(\log D^{L}\right) \longrightarrow 0,
$$

and the induced natural map,

$$
\left.\Omega_{L}^{p}\left(\log D^{L}\right) \longrightarrow \Omega_{X}^{p+1}(\log D)\right|_{L} \otimes \omega_{L / X},
$$

where $\omega_{L / X} \simeq \mathcal{L}_{L}$ as in [17].

Through the rest of this section all morphisms between sheaves and complexes are meant to be in $D(X)$ even if only sheaves are involved. Let $l: L \rightarrow X$ be the embedding of $L$ into $X$. The definition of $l$ for a finite morphism [16, VI 3.1, p. 311, p. 165] together with the fundamental local isomorphism [16, III 7.2] shows that

$$
\left.l^{\prime} \Omega_{X}^{p}(\log D) \simeq_{\mathrm{qis}} \Omega_{X}^{p}(\log D)\right|_{L} \otimes \omega_{L / X}[-1],
$$

and then the trace map for residual complexes gives

$$
\operatorname{Tr}_{l}: R l_{*}\left(\left.\Omega_{X}^{p}(\log D)\right|_{L} \otimes \omega_{L / X}\right)[-1] \longrightarrow \Omega_{X}^{p}(\log D) .
$$

Combining (3.1.1.1) and (3.1.1.2) one has:

$$
R_{l_{*}} \Omega_{L}^{p}\left(\log D^{L}\right)[-1] \longrightarrow \Omega_{X}^{p+1}(\log D) .
$$

Note, that the left-hand side is supported on $L$, so the map factors through $R \mathcal{H}_{L} \Omega_{X}^{p+2}(\log D)$. Also, by the proof of [17, II.2.2] this map is compatible with the differential of the de Rham complex, so by taking the above for all $p$ one has a trace map:

$$
\operatorname{Tr}_{L}: R l_{*} \Omega_{L}\left(\log D^{L}\right)[-2] \longrightarrow R \mathcal{H}_{L} \Omega_{X}(\log D) .
$$

LEMMA 3.1.2 [17, II.3.1]. $\operatorname{Tr}_{\mathrm{L}}$ in (3.1.1.3) is a quasi-isomorphism.

Remark 3.1.2. The proof of this lemma is taken from [17, II.3.1] with some small modifications and repeating it for logarithmic differentials instead of ordinary ones. It is included for the benefit of the reader as this constitutes an important step in the entire proof.

Proof. Since $L$ is of codimension one and $\Omega_{X}^{p}(\log D)$ is locally free for all $p$, there is only one nonzero local cohomology sheaf, namely $\mathcal{H}_{L}^{1} \Omega_{X}^{p}(\log D)$. Furthermore, $\mathcal{H}_{L}^{1} \Omega_{X}^{p}(\log D)$ can be identified with $\left.\Omega_{X}^{p}(\log D)\right|_{X \backslash L} / \Omega_{X}^{p}(\log D)$, locally isomorphic to $\Omega_{X}^{p}(\log D)\left[f^{-1}\right] / \Omega_{X}^{p}(\log D)$ where $f$ is a local equation of $L$ in $X$. Therefore,

$$
R \mathcal{H}_{L} \Omega_{X}(\log D) \simeq_{\text {qis }} \mathcal{H}_{L}^{1} \Omega_{X}(\log D)[-1]
$$


where $\mathcal{H}_{L}^{1} \Omega_{X}(\log D)$ denotes the complex whose $p$ th term is $\mathcal{H}_{L}^{1} \Omega_{X}^{p}(\log D)$ and whose differential is the one induced from the differential of the de Rham complex.

Using the local isomorphism $\mathcal{H}_{L}^{1} \Omega_{X}^{p}(\log D) \simeq \Omega_{X}^{p}(\log D)\left[f^{-1}\right] / \Omega_{X}^{p}(\log D)$ one sees easily that $\operatorname{Tr}_{L}$ is induced by the map

$$
\Omega_{L}^{p}\left(\log D^{L}\right)[-1] \longrightarrow \mathcal{H}_{L}^{1} \Omega_{X}^{p}(\log D),
$$

given by $\eta \mapsto \eta \wedge f^{-1} d f$, where $\eta \in \Omega_{L}^{p}\left(\log D^{L}\right)$.

Next assume that $L=\operatorname{Spec} A$ and $X=\operatorname{Spec} B$ are affine. Let $f \in B$ be an equation of $L$ in $X$, so $A \simeq B /(f)$. It is enough to prove the desired quasi-isomorphism after passing to the completion with respect to the $f$-adic topology (cf. [17, p.38]), so we may assume, that $B \simeq A[[f]]$ by [17, II.1.2].

Based on the above discussion, it will be sufficient to show that the map,

$$
\tau: \Omega_{A}\left(\log D^{L}\right)[-1] \longrightarrow \Omega_{B}(\log D)\left[f^{-1}\right] / \Omega_{B}(\log D),
$$

given by $\eta \mapsto \eta \wedge f^{-1} \mathrm{~d} f$ for $\eta \in \Omega_{L}^{p}\left(\log D^{L}\right)$ is a quasi-isomorphism of complexes.

Let $\gamma \in \Omega_{B}^{p+1}(\log D)\left[f^{-1}\right] / \Omega_{B}^{p+1}(\log D)$. Then $\gamma$ can be written as $\gamma=\gamma_{1}+\gamma_{2} \wedge \mathrm{d} f$, where $\gamma_{1} \in \Omega_{B}^{p+1}(\log D)\left[f^{-1}\right]$ and $\gamma_{2} \in \Omega_{B}^{p}(\log D)\left[f^{-1}\right]$, such that $\gamma_{i}=\sum_{j=0}^{k} \gamma_{i j} f^{-j}$ for $i=1,2$ and a suitable $k \in \mathbb{N}$ with $\gamma_{i j} \in \Omega_{B}^{p+2-i}(\log D)$. Furthermore, using the fact that $B \simeq A[[f]], \gamma_{i j}=\sum_{n=0}^{\infty} \gamma_{i j n} f^{n}$, where $\gamma_{i j n} \in \Omega_{A}^{p+2-i}\left(\log D^{L}\right)$. Hence,

$$
\gamma=\sum_{j=0}^{k} \sum_{n=0}^{\infty}\left(\gamma_{1 j n}+\gamma_{2 j n} \wedge \mathrm{d} f\right) f^{n-j}
$$

Notice that all but a finite number of terms of this expression will be in $\Omega_{B}^{p+1}(\log D)$, so one obtains that $\gamma$ can be written uniquely in the form

$$
\gamma=\sum_{s=1}^{N}\left(\alpha_{s}+\beta_{s} \wedge \mathrm{d} f\right) f^{-s}
$$

for a suitable $N \in \mathbb{N}$ and $\alpha_{s} \in \Omega_{A}^{p+1}\left(\log D^{L}\right)$ and $\beta_{s} \in \Omega_{A}^{p}\left(\log D^{L}\right)$.

Now $d \gamma=0$ if and only if

$$
\begin{aligned}
\mathrm{d} \alpha_{s} & =0, \quad s=1, \ldots, N, \\
\mathrm{~d} \beta_{1} & =0, \\
\mathrm{~d} \beta_{s+1} & =(-1)^{p+1} s \alpha_{s}, \quad s=1, \ldots, N .
\end{aligned}
$$

Let

$$
\theta=(-1)^{p+1} \sum_{s=2}^{N} \frac{1}{s-1} \beta_{s} f^{-s+1} \in \Omega_{A}^{p}\left(\log D^{L}\right) .
$$

Then $\gamma=\mathrm{d} \theta+\beta_{1} \wedge f^{-1} \mathrm{~d} f$ where $\mathrm{d} \beta_{1}=0$. Hence, $h^{i}(\tau)$ is surjective for all $i$.

Finally if $\beta_{1} \wedge f^{-1} \mathrm{~d} f=\mathrm{d} \gamma^{\prime}$ for some $\gamma^{\prime} \in \Omega_{B}^{p}(\log D)\left[f^{-1}\right] / \Omega_{B}^{p}(\log D)$, then a similar expression for $\gamma^{\prime}$ as the one for $\gamma$ in (3.1.2.3) shows that then there exists a $\rho \in \Omega_{A}^{p-1}\left(\log D^{L}\right)$ such that $\gamma^{\prime}=\rho \wedge f^{-1} \mathrm{~d} f$. Therefore, $h^{i}(\tau)$ is also injective for all $i$. 


\subsection{STRONG AMPLENESS}

In this subsection the extra assumptions made in the previous subsection are dropped, in particular $X$ is not necessarily smooth, but (2.1) is still in effect.

DEFINITION 3.2.1. Let $\mathcal{K}$ be a semi-ample line bundle on $X$. Then $\mathcal{K}$ is called strongly ample with respect to $X \backslash D$ if it is ample with respect to $X \backslash D$ and there exists a proper birational morphism, $\alpha: \tilde{X} \rightarrow X$, such that for $\tilde{D}=\alpha^{*} D$, $\alpha_{\tilde{X} \backslash \tilde{D}}: \tilde{X} \backslash \tilde{D} \rightarrow X \backslash D$ is an isomorphism and there exists an effective divisor $\tilde{B}$ on $\tilde{X}$ such that supp $\tilde{B}=\operatorname{supp} \tilde{D}$ and $\alpha^{*} \mathcal{K}^{a}(\tilde{B})$ is an ample line bundle for some $a>0$.

In particular, if $\tau \in H^{0}\left(X, \mathcal{K}^{a m}\right)$. is a general section for some $m \gg 0$ and $K=(\tau=0)$, then $\alpha^{*} K+m \tilde{B}$ is an effective ample Cartier divisor supported on $\tilde{D} \cup \alpha^{*} K$, hence $\tilde{X} \backslash\left(\tilde{D} \cup \alpha^{*} K\right) \simeq X \backslash(D \cup K)$ is affine.

3.2.2. Note that if $D=\emptyset$, then $\mathcal{K}$ is strongly ample if and only if it is ample. It is also clear that if $\mathcal{K}$ is strongly ample then it is also big. On the other hand, let $\pi: X \rightarrow \mathbb{P}^{n}$ be the blow up of $\mathbb{P}^{n}$ at a single point for $n \geqslant 2$. Let $D$ be the exceptional divisor of $\pi$. Then $\pi^{*} \mathcal{O}_{X}(1)$ is semi-ample and big, but not strongly ample with respect to $X \backslash D$.

It will be very important in Section 4 that this property is inherited by restrictions to $L$ :

LEMMA 3.2.3. If $\mathcal{K}$ is strongly ample with respect to $X \backslash D$, then $\mathcal{K}_{L}=\left.\mathcal{K}\right|_{L}$ is strongly ample with respect to $L \backslash D^{L}$.

Proof. $\mathcal{K}$ is ample with respect to $X \backslash D$, so $\mathcal{K}_{L}$ is ample with respect to $(X \backslash D) \cap L=L \backslash D^{L}$. Let $\alpha: \tilde{X} \rightarrow X$ be a proper birational morphism, $\tilde{D}=\alpha^{*} D$, and $\tilde{B}$ an effective divisor on $\tilde{X}$ such that supp $\tilde{B}=\operatorname{supp} \tilde{D}$ and $\alpha^{*} \mathcal{K}^{a}(\tilde{B})$ is ample for some $a>0$.

Let $\tilde{L}$ be the proper transform of $L$ on $\tilde{X}, \alpha_{L}=\left.\alpha\right|_{\tilde{L}}$ and $\tilde{B}^{L}=\left.\tilde{B}\right|_{L}$. It is easy to see that $\mathcal{K}_{L}$ and $\alpha_{L}: \tilde{L} \rightarrow L$ satisfies the requirements of Definition 3.2.1.

The following lemma gives important examples for strongly ample line bundles.

LEMMA 3.2.4. Assume that there is an effective $\mathbb{Q}$-Cartier divisor B, such that supp $B=\operatorname{supp} D$ and, in addition to (2.1), one of the following holds:

(3.2.4.1) $\mathcal{L}$ is ample, or

(3.2.4.2) B is nef.

Then $\mathcal{M}$ is strongly ample with respect to $X \backslash D$.

Proof. It is enough to prove that $\mathcal{L}=\mathcal{M}^{N}$ is strongly ample with respect to $X \backslash D$.

If $\mathcal{L}$ is ample and $B$ is $\mathbb{Q}$-Cartier, then $\mathcal{L}^{a}(b B)$ is ample for some $a, b>0$. 
In the case of (3.2.4.2), let $\phi: X \rightarrow Z$ be the morphism given by the global sections of $\mathcal{L}$ and $\mathcal{A}$ an ample line bundle on $Z$, such that $\mathcal{L}=\phi^{*} \mathcal{A}$. Further let $\alpha: \tilde{X} \rightarrow X$ be the blowing up of $X$ along the exceptional set of $\phi$ and $\tilde{D}=\alpha^{*} D$. Note that the exceptional set of $\tilde{\phi}=\phi \circ \alpha$ is a Cartier divisor with support contained in $\operatorname{supp} \tilde{D}=\operatorname{supp} \alpha^{*} B$. Now there exists a $\tilde{\phi}$-exceptional divisor $E$ on $\tilde{X}$, such that $\tilde{\phi}^{*} \mathcal{A}^{a}(-E)=\alpha^{*} \mathcal{L}^{a}(-E)$ is ample for some $a>0$. Since $E$ is $\tilde{\phi}$-exceptional, supp $E \subseteq \operatorname{supp} \tilde{D}=\operatorname{supp} \alpha^{*} B$, so for some $b>0, \tilde{B}=b \alpha^{*} B-E$ is effective and $\operatorname{supp} \tilde{B}=\operatorname{supp} \tilde{D}$. Finally, $\alpha^{*} \mathcal{L}^{a}(\tilde{B})$ is ample, since $B$ is nef.

\subsection{GYSIN MORPHISM}

3.3.1. Using the notation and assumptions of (2.1) further assume that $\mathcal{M}$ is strongly ample with respect to $X \backslash D$.

3.3.2. Again, $\varepsilon .:(X, D.) \rightarrow(X, D)$ denotes a good hyperresolution. Applying (3.1.2) for $l^{i}: L_{i} \hookrightarrow X_{i}$ one obtains the following natural quasi-isomorphism

$$
R l_{*}^{i} \Omega_{L_{i}}\left(\log D_{i}^{L_{i}}\right)[-2] \stackrel{\simeq_{\mathrm{qis}}}{\longrightarrow} R \mathcal{H}_{L_{i}} \Omega_{X_{i}}\left(\log D_{i}\right) .
$$

By (2.1.1), this implies that there exists a quasi-isomorphism

$$
R l_{*} \underline{\Omega}_{L}\left(\log D^{L}\right)[-2] \stackrel{\simeq_{\text {qis }}}{\longrightarrow} R \mathcal{H}_{L} \underline{\Omega}_{X}(\log D) .
$$

Let $j: X \backslash D \rightarrow X$ and $j^{L}: L \backslash D^{L} \rightarrow L \rightarrow X$ be the inclusion maps. Then by (1.2.1) (3.3.2.1) gives a quasi-isomorphism

$$
R j_{*}^{L} \mathbb{C}_{L \backslash D^{L}}[-2] \stackrel{\simeq_{\text {qis }}}{\longrightarrow} R \mathcal{H}_{L} R j_{*} \mathbb{C}_{X \backslash D} \simeq_{\mathrm{qis}} R \mathcal{H}_{L \backslash D^{L}} \mathbb{C}_{X \backslash D} .
$$

Applying $R \Gamma$ to both sides one obtains a quasi-isomorphism

$$
R \Gamma \mathbb{C}_{L \backslash D^{L}}[-2] \stackrel{\simeq_{\mathrm{qis}}}{\longrightarrow} R \Gamma_{L \backslash D^{L}} \mathbb{C}_{X \backslash D}
$$

In particular

$$
H^{i-2}\left(L \backslash D^{L}, \mathbb{C}\right) \stackrel{\simeq}{\longrightarrow} H_{L \backslash D^{L}}^{i}(X \backslash D, \mathbb{C})
$$

is an isomorphism for all $i$. On the other hand,

$$
R \Gamma_{L \backslash D^{L}} \mathbb{C}_{X \backslash D} \longrightarrow R \Gamma \mathbb{C}_{X \backslash D} \longrightarrow R \Gamma \mathbb{C}_{X \backslash(D \cup L)} \stackrel{+1}{\longrightarrow}
$$

forms a distinguished triangle. By (3.2.1) $\quad X \backslash(D \cup L)$ is affine, so $H^{j}(X \backslash(D \cup L), \mathbb{C})=0$ for $j>\operatorname{dim} X$ cf. [17, II.4.6], [13], [15, III.3.1(i)]. Hence,

$$
H_{L \backslash D^{L}}^{i}(X \backslash D, \mathbb{C}) \rightarrow H^{i}(X \backslash D, \mathbb{C})
$$

is an isomorphism for $i>\operatorname{dim} X+1$ and surjective for $i=\operatorname{dim} X+1$. Combining 
this with (3.3.2.2) one obtains that

$$
H^{i-2}\left(L \backslash D^{L}, \mathbb{C}\right) \longrightarrow H^{i}(X \backslash D, \mathbb{C})
$$

is an isomorphism for $i>\operatorname{dim} X+1$ and surjective for $i=\operatorname{dim} X+1$. Furthermore, by the construction of these maps it is clear that they respect the Hodge decomposition (1.2.4). Therefore,

$$
G: \mathbb{H}^{q-1}\left(L, \underline{\Omega}_{L}^{p-1}\left(\log D^{L}\right)\right) \longrightarrow \mathbb{H}^{q}\left(X, \underline{\Omega}_{X}^{p}(\log D)\right)
$$

is an isomorphism for $p+q>\operatorname{dim} X+1$ and surjective for $p+q=\operatorname{dim} X+1$.

\section{Logarithmic Vanishing Theorem}

THEOREM 4.1. Let $X$ be a projective variety and $D$ an effective divisor on $X$. Let $\mathcal{M}$ be a semi-ample line bundle on $X$ that is strongly ample with respect to $X \backslash D$. Then for $p+q>n$,

$\mathbb{H}^{q}\left(X, \underline{\Omega}_{X}^{p}(\log D) \otimes \mathcal{M}=0\right.$.

Proof. Using the notation and assumptions of (2.1), we first prove the same statement for $\mathcal{L}=\mathcal{M}^{N}, N \gg 0$ :

4.1.1. $\mathbb{H}^{q}\left(X, \underline{\Omega}_{X}^{p}(\log D) \otimes \mathcal{L}\right)=0 \quad$ for $p+q>\operatorname{dim} X$.

Tensoring the short exact sequence,

$$
0 \longrightarrow \mathcal{O}_{X} \longrightarrow \mathcal{L} \longrightarrow \mathcal{L}_{L} \longrightarrow 0,
$$

by $\underline{\Omega}_{X}^{p}(\log D)$ leads to the distinguished triangle,

$$
\underline{\Omega}_{X}^{p}(\log D) \longrightarrow \underline{\Omega}_{X}^{p}(\log D) \otimes \mathcal{L} \longrightarrow \underline{\Omega}_{X}^{p}(\log D) \otimes \otimes^{L} \mathcal{L}_{L} \stackrel{+1}{\longrightarrow}
$$

and the corresponding long exact hypercohomology sequence:

$$
\mathbb{H}^{q-1}\left(L, \underline{\Omega}_{X}^{p}(\log D) \otimes{ }^{L} \mathcal{L}_{L}\right) \stackrel{\partial}{\longrightarrow} \mathbb{H}^{q}\left(X, \underline{\Omega}_{X}^{p}(\log D)\right) \longrightarrow \mathbb{H}^{q}\left(X, \underline{\Omega}_{X}^{p}(\log D) \otimes \mathcal{L}\right) .
$$

On the other hand, Lemma 2.2 gives the distinguished triangle,

$$
\underline{\Omega}_{L}^{p-1}\left(\log D^{L}\right) \longrightarrow \underline{\Omega}_{X}^{p}(\log D) \otimes^{L} \mathcal{L}_{L} \longrightarrow \underline{\Omega}_{L}^{p}\left(\log D^{L}\right) \otimes \mathcal{L}_{L} \stackrel{+1}{\longrightarrow},
$$

and in turn the long exact hypercohomology sequence:

$$
\begin{aligned}
\mathbb{H}^{q-1}\left(L, \underline{\Omega}_{L}^{p-1}\left(\log D^{L}\right)\right) & \longrightarrow \mathbb{H}^{q-1}\left(L, \underline{\Omega}_{X}^{p}(\log D) \otimes \mathcal{L}_{L}\right) \\
& \longrightarrow \mathbb{H}^{q-1}\left(L, \underline{\Omega}_{L}^{p}\left(\log D^{L}\right) \otimes \mathcal{L}_{L}\right) .
\end{aligned}
$$


Now by induction and Lemma 3.2.3 we may assume that $\mathbb{H}^{q-1}\left(L, \underline{\Omega}_{L}^{p}\left(\log D^{L}\right) \otimes \mathcal{L}_{L}\right)=0$. Hence,

$$
\phi: \mathbb{H}^{q-1}\left(L, \underline{\Omega}_{L}^{p-1}\left(\log D^{L}\right)\right) \longrightarrow \mathbb{H}^{q-1}\left(L, \underline{\Omega}_{X}^{p}(\log D) \otimes^{L} \mathcal{L}_{L}\right)
$$

is an isomorphism for $p+q>\operatorname{dim} X+1$ and surjective for $p+q=\operatorname{dim} X+1$. Furthermore $\phi$ is induced by the map $\underline{\Omega}_{L}^{p-1}\left(\log D^{L}\right) \longrightarrow \underline{\Omega}_{X}^{p}(\log D) \otimes \mathcal{L}_{L}$ which locally is given by $\eta \rightarrow \eta \wedge \mathrm{d} f \otimes f^{-1}$ where $f$ is a local equation of $L$ in $X$. So $\phi$ is defined the same way as the Gysin map was, hence, the following diagram is commutative, where $G$ is from (3.3.2.3) and $\partial$ is from (4.1.2).

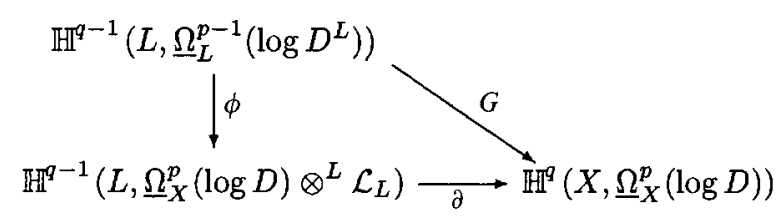

Now $G$ and $\phi$ are isomorphisms for $p+q>\operatorname{dim} X+1$ and surjective for $p+q=\operatorname{dim} X+1$, so the same holds for $\partial$. However, then (4.1.2) implies that

$$
\mathbb{H}^{q}\left(X, \underline{\Omega}_{X}^{p}(\log D) \otimes \mathcal{L}\right)=0 \quad \text { for } p+q>\operatorname{dim} X .
$$

To obtain the statement in the general case one uses the usual covering trick:

\subsubsection{Let}

$$
\pi: \tilde{X}=\operatorname{Spec}_{X} \bigoplus_{i=0}^{N-1} \mathcal{M}^{-i} \longrightarrow X
$$

be the cover obtained by taking the $N^{\text {th }}$-root of $L$. Now the trace map of $\pi$ provides a left inverse to the natural map, cf. [15, p. 151], [11, 3.22]:

$$
\underline{\Omega}_{X}^{p}(\log D) \longrightarrow R \pi_{*} \underline{\Omega}_{\tilde{X}}^{p}\left(\log \pi^{*} D\right)
$$

Applying $\mathbb{H}^{q}$ and using (4.1.1) on $\tilde{X}$ proves the statement.

COROLLARY 4.2 [14]. Let $Y$ be a smooth complex projective variety and $\mathcal{M}$ a semi-ample and big line bundle on $Y$. Then

$$
H^{i}\left(Y, \omega_{Y} \otimes \mathcal{M}\right)=0 \quad \text { for } i>0 .
$$

Proof. First assume that $\mathcal{M}$ is generated by global sections. Let $\phi: Y \rightarrow X$ be the morphism given by the global sections of $\mathcal{M}$. Then there exists an ample line bundle, $\mathcal{L}$, on $X$ such that $\mathcal{M}=\phi^{*} \mathcal{L}$, so by (1.2.8), Lemma 3.2.4, and Theorem 4.1

$$
H^{i}\left(Y, \omega_{Y} \otimes \mathcal{M}\right) \simeq \mathbb{H}^{i}\left(X, R \phi_{*} \omega_{Y} \otimes \mathcal{L}\right) \simeq \mathbb{H}^{i}\left(X, \underline{\Omega}_{X}^{n}(\log \emptyset) \otimes \mathcal{L}\right)=0 .
$$

The general case is now proved by the usual covering trick, cf. Theorem 4.1. 
We also have a relative version:

THEOREM 4.3. Let $\psi: X \rightarrow Z$ be a projective morphism and $D$ an effective $\mathbb{Q}$-Cartier divisor on $X$. Let $\mathcal{L}$ be a $\psi$-ample line bundle on $X$. Then for $p+q>n$,

$$
R^{q} \psi_{*}\left(\underline{\Omega}_{X}^{p}(\log D) \otimes \mathcal{L}\right)=0 .
$$

Proof. The statement is local, so we may assume that $Z$ is projective. Let $\mathcal{M}$ be an ample line bundle on $Z$, such that for all $p, q, R^{q} \psi_{*}\left(\underline{\Omega}_{X}^{p}(\log D) \otimes \mathcal{L}\right) \otimes \mathcal{M}$ is generated by global sections and have no higher cohomology. This can be done because $\underline{\Omega}_{X}^{p}(\log D)$ is bounded and has coherent cohomology sheaves. Furthermore, choose $\mathcal{M}$ in such a way, that $\mathcal{L} \otimes \psi^{*} \mathcal{M}$ is ample on $X$. Then by the Leray spectral sequence, Lemma 3.2.4 and Theorem 4.1,

$$
H^{0}\left(Z, R^{q} \psi_{*}\left(\underline{\Omega}_{X}^{p}(\log D) \otimes \mathcal{L}\right) \otimes \mathcal{M}\right)=\mathbb{H}^{q}\left(X, \underline{\Omega}_{X}^{p}(\log D) \otimes \mathcal{L} \otimes \psi^{*} \mathcal{M}\right)=0 .
$$

Since $R^{q} \psi_{*}\left(\underline{\Omega}_{X}^{p}(\log D) \otimes \mathcal{L}\right) \otimes \mathcal{M}$ is generated by global sections, this proves the statement.

Finally, this gives a bound on the range of degrees where $\underline{\Omega}_{X}^{p}(\log D)$ can have nonzero cohomology sheaves.

COROLARY 4.4. Let $X$ be a projective variety and $D$ an effective $\mathbb{Q}$-Cartier divisor on $X$. Then $h^{q}\left(\underline{\Omega}_{X}^{p}(\log D)\right)=0$ for $q>n-p$ or $q<0$.

Proof. Let $\psi=\operatorname{id}_{X}: X \rightarrow X$ and $\mathcal{M}=\mathcal{O}_{X}$. The second inequality is simply (1.2.5).

Regarding the case of $H^{q}\left(Y, \Omega_{Y}^{p} \otimes \mathcal{M}\right)$ for $p<n$, Ramanujam has already noticed that if $\mathcal{M}$ is only semi-ample (or even generated by global sections) and big, than vanishing does not necessarily hold [35]. However, since globally generated and big line bundles are pull-backs of (very) ample ones, Theorem 4.1 can be considered as a substitute. Later applications will show that it can actually be used for this purpose.

\section{Relative Complexes}

Let $f: X \rightarrow C$ be a morphism such that $C$ is a smooth complex curve. Let $\Delta \subseteq C$ be a finite set and $D=f^{*} \Delta$. Let $\varepsilon:(X$., $D.) \longrightarrow(X, D)$ be a good hyperresolution, and consider the map $f_{i}=f \circ \varepsilon_{i}: X_{i} \longrightarrow C$. The goal is to construct a complex whose cohomological properties resemble those of $\Omega_{X / C}^{p}$ in the smooth case.

Taking the wedge product induces a map,

$$
\Omega_{X_{i}}^{p}\left(\log D_{i}\right) \otimes f_{i}^{*} \omega_{C}(\Delta) \longrightarrow \Omega_{X_{i}}^{p+1}\left(\log D_{i}\right)
$$


This is obviously compatible with $\varepsilon$, so it gives a morphism of complexes:

$$
\wedge_{p}: \underline{\Omega}_{X}^{p}(\log D) \otimes f^{*} \omega_{C}(\Delta) \longrightarrow \underline{\Omega}_{X}^{p+1}(\log D) .
$$

It is also easy to see that this is independent of the actual hyperresolution used cf. [26, p. 375]. Hence, $\wedge_{p}$ is a well-defined natural map in $D(X)$.

Choose a representative, $K_{p} \in \mathcal{O} b j(C(X))$, of $\underline{\Omega}_{X}^{p}(\log D)$ for all $p$ such that $\wedge_{p}$ is represented by morphisms $K_{p} \rightarrow K_{p+1}$ in $\mathcal{M o r}(C(X))$. By abuse of notation this will also be denoted by $\wedge_{p}$. Let $\wedge_{p}^{\prime}=\wedge_{p} \otimes \operatorname{id}_{f^{*} \omega_{C}(\Delta)} \in \operatorname{Hom}_{C(X)}\left(\left(K_{p} \otimes f^{*} \omega_{C}(\Delta)\right.\right.$, $\left.K_{p+1} \otimes f^{*} \omega_{C}(\Delta)\right)$. Since $\omega_{C}(\Delta)$ is a line bundle, $\wedge_{p} \circ \wedge_{p-1}^{\prime}=0$. Let $M_{r}=0 \in \mathcal{O} b j(C(X)), \quad w_{r}^{\prime \prime}=0 \in \operatorname{Hom}_{C(X)}\left(K_{r} \otimes f^{*} \omega_{C}(\Delta), \quad M_{r} \otimes f^{*} \omega_{C}(\Delta)\right) \quad$ and $w_{r}^{\prime}=0 \in \operatorname{Hom}_{C(X)}\left(M_{r} \otimes f^{*} \omega_{C}(\Delta), K_{r+1}\right)$ for $r \geqslant n$. Assume that $p<n$ and for every $q>p, M_{q} \in \mathcal{O} b j(C(X))$ is defined. Assume further that there are morphisms of complexes,

$$
w_{q}^{\prime \prime}: K_{q} \otimes f^{*} \omega_{C}(\Delta) \longrightarrow M_{q} \otimes f^{*} \omega_{C}(\Delta) \quad \text { and } \quad w_{q}^{\prime}: M_{q} \otimes f^{*} \omega_{C}(\Delta) \longrightarrow K_{q+1},
$$

such that

$$
\wedge_{q}=w_{q}^{\prime} \circ w_{q}^{\prime \prime} \quad \text { and } \quad w_{q}^{\prime \prime} \circ \wedge_{q-1}^{\prime}=0 .
$$

Let

$$
w_{q}=w_{q}^{\prime \prime} \otimes \operatorname{id}_{f^{*} \omega_{C}(\Delta)^{-1}}: K_{r} \longrightarrow M_{r}
$$

and

$$
M_{p}=M\left(w_{p+1}\right)[-1] \otimes f^{*} \omega_{C}(\Delta)^{-1} \in \mathcal{O} b j(C(X)),
$$

i.e.,

$$
M_{p}^{m} \otimes f^{*} \omega_{C}(\Delta)=K_{p+1}^{m} \oplus M_{p+1}^{m-1}
$$

and

$$
d_{M_{p} \otimes f^{*} \omega_{C}(\Delta)}^{m}=\left(\begin{array}{cc}
d_{K_{p+1}}^{m} & 0 \\
-w_{p+1}^{m} & -d_{M_{p+1}}^{m-1}
\end{array}\right)
$$

Also let

$$
w_{p}^{\prime \prime}=\left(\begin{array}{c}
\wedge_{p} \\
0
\end{array}\right): K_{p} \otimes f^{*} \omega_{C}(\Delta) \longrightarrow M_{p} \otimes f^{*} \omega_{C}(\Delta)
$$

and

$$
w_{p}^{\prime}=\left(\operatorname{id}_{K_{p+1}}, 0\right): M_{p} \otimes f^{*} \omega_{C}(\Delta) \longrightarrow K_{p+1} .
$$

$w_{p}^{\prime}$ is a morphism of complexes by the definition of the mapping cone and $w_{p}^{\prime}$ is a morphism of complexes because $w_{p+1} \circ \wedge_{p}=0$. It is also obvious that $\wedge_{p}=w_{p}^{\prime} \circ w_{p}^{\prime}$ and $w_{p}^{\prime} \circ \wedge_{p-1}^{\prime}=0$. 
By their definition, the equivalence classes of $w_{p}, w_{p}^{\prime}$ and $w_{p}^{\prime \prime}$ in $D(X)$ are independent of the hyperresolution chosen. From now on these symbols will denote their equivalence classes in $D(X)$. A map will mean an element of $\operatorname{Mor}(D(X))$, so it is possibly not represented by an actual morphism of complexes between two arbitrary representatives of the respective objects.

THEOREM-DEFINITION 5.1. Let $f: X \rightarrow C$ be a morphism between complex varieties such that $\operatorname{dim} X=n$ and $C$ is a smooth curve. Let $\Delta \subseteq C$ be a finite set and $D=f^{*} \Delta$. For every nonnegative integer $p$ there exists a complex $\underline{\Omega}_{X / C}^{p}(\log D) \in \mathcal{O} b j(D(X))$ with the following properties.

(5.1.1) The natural map $\wedge_{p}$ factors through $\underline{\Omega}_{X / C}^{p}(\log D) \otimes f^{*} \omega_{C}(\Delta)$, i.e., there exist maps:

$$
\begin{aligned}
& w_{p}^{\prime \prime}: \underline{\Omega}_{X}^{p}(\log D) \otimes f^{*} \omega_{C}(\Delta) \longrightarrow \underline{\Omega}_{X / C}^{p}(\log D) \otimes f^{*} \omega_{C}(\Delta) \quad \text { and } \\
& w_{p}^{\prime}: \underline{\Omega}_{X / C}^{p}(\log D) \otimes f^{*} \omega_{C}(\Delta) \longrightarrow \underline{\Omega}_{X}^{p+1}(\log D)
\end{aligned}
$$

such that $\wedge_{p}=w_{p}^{\prime} \circ w_{p}^{\prime \prime}$

(5.1.2) If $w_{p}=w_{p}^{\prime \prime} \otimes \operatorname{id}_{f^{*} \omega_{C}(\Delta)^{-1}}: \underline{\Omega}_{X}^{p}(\log D) \longrightarrow \underline{\Omega}_{X / C}^{p}(\log D)$, then

$$
\underline{\Omega}_{X / C}^{p}(\log D) \otimes f^{*} \omega_{C}(\Delta) \stackrel{w_{p}^{\prime}}{\longrightarrow} \underline{\Omega}_{X}^{p+1}(\log D) \stackrel{w_{p+1}}{\longrightarrow} \underline{\Omega}_{X / C}^{p+1}(\log D) \stackrel{+1}{\longrightarrow}
$$

is a distinguished triangle in $D(X)$.

(5.1.3) $\quad w_{p}$ is functorial, i.e., if $\phi: Y \rightarrow X$ is a $C$-morphism, then there are natural maps in $D(X)$ forming a commutative diagram:

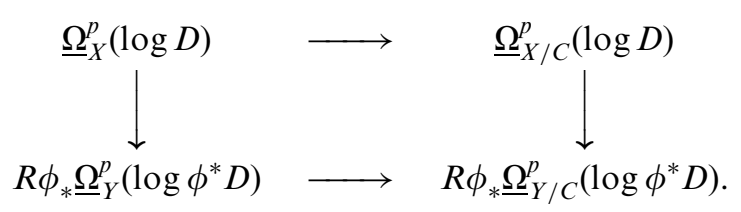

(5.1.4) If $f$ is smooth over $C \backslash \Delta$ then $\underline{\Omega}_{X / C}^{p}(\log D) \simeq_{\mathrm{qis}} \Omega_{X / C}^{p}(\log D)$.

(5.1.5) $\underline{\Omega}_{X / C}^{r}(\log D)=0$ for $r \geqslant n$ and if $f$ is proper, then $\underline{\Omega}_{X / C}^{p}(\log D) \in$ $\mathcal{O} b j\left(D_{\text {coh }}^{b}(X)\right)$ for every $p$.

Proof. Let $\underline{\Omega}_{X / C}^{p}(\log D) \simeq_{\text {qis }}\left[M_{p}\right] \in \mathcal{O b j}(D(X))$. Then (5.1.1), (5.1.2) and the first part of (5.1.5) follows from the discussion above. Using (5.1.2), the first part of (5.1.5) and descending induction on $p,(5.1 .3),(5.1 .4)$ and the rest of (5.1.5) follows from (1.2.2) and (1.2.7).

Note that the combination of (1.2.2), (1.2.6), and (5.1.3) implies that if $\phi: Y \rightarrow X$ is a C-morphism, then there are natural maps in $D(X)$ forming a commutative 
diagram:

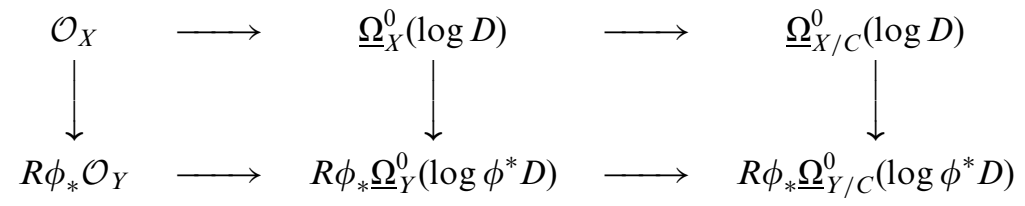

\section{More vanishing theorems}

THEOREM 6.1. Let $X$ be a projective variety of dimension $n$ and $f: X \rightarrow C$ a morphism to a smooth complex curve. Let $\Delta \subseteq C$ be a finite set and $D=f^{*} \Delta$. Let $\mathcal{L}$ be a line bundle on $X$ such that $\mathcal{L}$ and $\mathcal{L} \otimes f^{*} \omega_{C}(\Delta)^{-(n-1)}$ are semi-ample and ample with respect to $X \backslash D$. Then

$$
\mathbb{H}^{n}\left(X, \underline{\Omega}_{X / C}^{0}(\log D) \otimes \mathcal{L} \otimes f^{*} \omega_{C}(\Delta)\right)=0 .
$$

Proof. Let $\mathcal{L}_{p}=\mathcal{L} \otimes f^{*} \omega_{C}(\Delta)^{-(p-1)}$ for $p=0, \ldots, n$. By assumption, $\mathcal{L}_{p}$ is semi-ample and ample with respect to $X \backslash D$ for $1 \leqslant p \leqslant n$ since either $f^{*} \omega_{C}(\Delta)$ or $f^{*} \omega_{C}(\Delta)^{-1}$ is semi-positive. In fact $\mathcal{L}_{p}$ is strongly ample with respect to $X \backslash D$ for $1 \leqslant p \leqslant n$ by Lemma 3.2.4 since $D$ is an effective, nef $\mathbb{Q}$-Cartier divisor. Twisting (5.1.2) by $\mathcal{L}_{p}$ yields the following distinguished triangle:

$$
\underline{\Omega}_{X / C}^{p-1}(\log D) \otimes \mathcal{L}_{p-1} \longrightarrow \underline{\Omega}_{X}^{p}(\log D) \otimes \mathcal{L}_{p} \longrightarrow \underline{\Omega}_{X / C}^{p}(\log D) \otimes \mathcal{L}_{p} \stackrel{+1}{\longrightarrow} .
$$

By Theorem $4.1 \mathbb{H}^{n-p(p-1)}\left(X, \underline{\Omega}_{X}^{p}(\log D) \otimes \mathcal{L}_{p}\right)=0$, so the map

$$
\mathbb{H}^{n-p}\left(X, \underline{\Omega}_{X / C}^{p}(\log D) \otimes \mathcal{L}_{p} \longrightarrow \mathbb{H}^{n-(p-1)}\left(X, \underline{\Omega}_{X / C}^{p-1}(\log D) \otimes \mathcal{L}_{p-1}\right)\right.
$$

is surjective for all $1 \leqslant p \leqslant n$. Observe that these maps form a chain as $p$ runs through $p=n, n-1, \ldots, 1$. So the composite map

$$
\mathbb{H}^{0}\left(X, \underline{\Omega}_{X / C}^{n}(\log D) \otimes \mathcal{L}_{n}\right) \longrightarrow \mathbb{H}^{n}\left(X, \underline{\Omega}_{X / C}^{0}(\log D) \otimes \mathcal{L}_{0}\right)
$$

is also surjective. However, $\underline{\Omega}_{X / C}^{n}(\log D)=0$ by construction (cf. (5.1.5)). Therefore the statement follows.

LEMMA 6.2. Let $\phi: Y \rightarrow X$ be a proper generically finite map of varieties of dimension $n$. Let $\mathcal{F}$ be a coherent sheaf on $Y$. Then the natural map $H^{n}\left(X, \phi_{*} \mathcal{F}\right) \rightarrow H^{n}(Y, \mathcal{F})$ is surjective.

Proof. Let $x \in X$ and let $d(x)=\operatorname{dim} Y_{x}$, the dimension of the fiber of $\phi$ over $x$. Now $\left(R^{j} \phi_{*} \mathcal{F}\right)_{x}=0$ for $j>d(x)$, so supp $R^{j} \phi_{*} \mathcal{F} \subseteq X_{j}=\{x \in X \mid d(x) \leqslant j\}$. Clearly, $X=X_{0} \cup X_{1} \cup \cdots \cup X_{n-1}$ and for all $j>0$, the dimension of $\phi^{-1}\left(X_{j}\right)$ is at most $n-1$, so $\operatorname{dim} X_{j}+j \leqslant n-1$. Hence, $\operatorname{dim} \operatorname{supp} R^{j} \phi_{*} \mathcal{F} \leqslant n-j-1$ for $j>0$. Therefore, $H^{i}\left(X, R^{j} \phi_{*} \mathcal{F}\right)=0$ for $j>0, i+j \geqslant n$. Finally this implies that in the 
Leray spectral sequence $H^{i}\left(X, R^{j} \phi_{*} \mathcal{F}\right) \Rightarrow H^{i+j}(Y, \mathcal{F})$ the only nonzero term for $i+j \geqslant n$ is $H^{n}\left(X, \phi_{*} \mathcal{F}\right)$.

LEMMA 6.3. Let $\phi: Y \rightarrow X$ be a proper generically finite map of normal varieties of dimension $n$. Let $\mathcal{L}$ be a line bundle on $X$.

(6.3.1) If $\phi$ is birational, then the natural map $H^{n}(X, \mathcal{L}) \rightarrow H^{n}\left(Y, \phi_{*} \mathcal{L}\right)$ is surjective.

(6.3.2) If $X$ is projective and has rational singularities, then the natural map $H^{n}(X, \mathcal{L}) \rightarrow H^{n}\left(Y, \phi^{*} \mathcal{L}\right)$ is injective.

Proof. If $\phi$ is birational, then $\mathcal{O}_{X} \simeq \phi_{*} \mathcal{O}_{Y}$, so $\mathcal{L} \simeq \phi_{*} \phi^{*} \mathcal{L}$, hence, Lemma 6.2 implies (6.3.1). If $X$ is projective and has rational singularities, then the natural map $\mathcal{O}_{X} \rightarrow R \phi_{*} \mathcal{O}_{Y}$ has a left inverse by [29, Theorem 2]. Hence, $H^{n}(X, \mathcal{L}) \rightarrow \mathbb{H}^{n}\left(X, R \phi_{*} \mathcal{O}_{Y} \otimes \mathcal{L}\right) \simeq H^{n}\left(Y, \phi^{*} \mathcal{L}\right)$ is injective.

THEOREM 6.4. Let $X$ be a projective variety of dimension $n$ and $f: X \rightarrow C$ a morphism to a smooth proper curve. Let $\Delta \subseteq C$ be a finite set and $D=f^{*} \Delta$. Assume that there exists a smooth projective variety, $Y$, and a proper generically finite map, $\phi: Y \rightarrow X$, such that $\left.h\right|_{Y \backslash B}: Y \backslash B \rightarrow C \backslash \Delta$ is smooth, where $h=f \circ \phi$ and $B=h^{*} \Delta$. Let $\tilde{C}$ be smooth proper curve, $\sigma: \tilde{C} \rightarrow C$ a finite cover, unramified over $C \backslash \Delta$. Assume that for $\tilde{\Delta}=\left(\sigma^{*} \Delta\right)_{\text {red }}, \omega_{\tilde{C}}(\tilde{\Delta}) \subset \sigma^{*} \omega_{C}(\Delta)$. Let $\tilde{X}$ be the normalization of $\tilde{C} \times_{C} X$ and $\tilde{Y} \rightarrow \tilde{C} \times_{C} Y$ a resolution of singularities such that it is an isomorphism over $\tilde{C} \backslash \sigma^{*} \Delta$ :

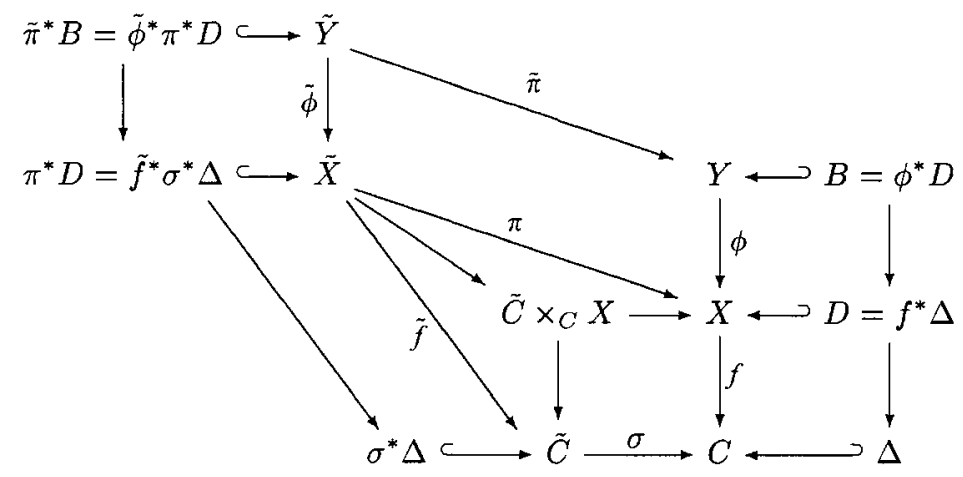

Assume that there exists a line bundle $\mathcal{L}$ on $X$ such that $\pi^{*} \mathcal{L}$ contains a line bundle $\tilde{\mathcal{L}}$ such that $\tilde{\mathcal{L}}$ and $\tilde{\mathcal{L}} \otimes \tilde{f}^{*} \omega_{C}(\tilde{\Delta})^{-(n-1)}$ are semi-ample and ample with respect to $\tilde{X} \backslash \pi^{*} D$.

(6.4.1) If $\phi$ is birational, then

$H^{n}\left(Y, \phi^{*} \mathcal{L} \otimes h^{*} \omega_{C}(\Delta)\right)=0$. 
(6.4.2) If $X$ has rational singularities, then

$$
H^{n}\left(X, \mathcal{L} \otimes f^{*} \omega_{C}(\Delta)\right)=0 .
$$

Proof. Let $\mathcal{M}=\mathcal{L} \otimes f^{*} \omega_{C}(\Delta)$ and $\tilde{\mathcal{M}}=\tilde{\mathcal{L}} \otimes \tilde{f}^{*} \omega_{\tilde{C}}(\tilde{\Delta}) \subseteq \pi^{*} \mathcal{M}$. By (5.1.6) one has the following commutative diagram:

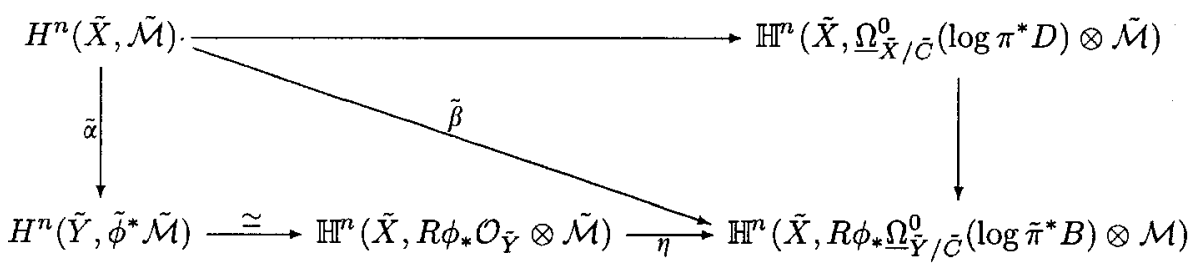

$\mathbb{H}^{n}\left(\tilde{X}, \underline{\Omega}_{\tilde{X} / \tilde{C}}^{0}\left(\log \pi^{*} D\right) \otimes \tilde{\mathcal{M}}\right)=0$ by Theorem 6.1 , so $\tilde{\beta}$ is the zero map. Furthermore $\eta$ is an isomorphism by (5.1.4), hence, $\tilde{\alpha}$ is the zero map as well.

Now consider the following commutative diagram:

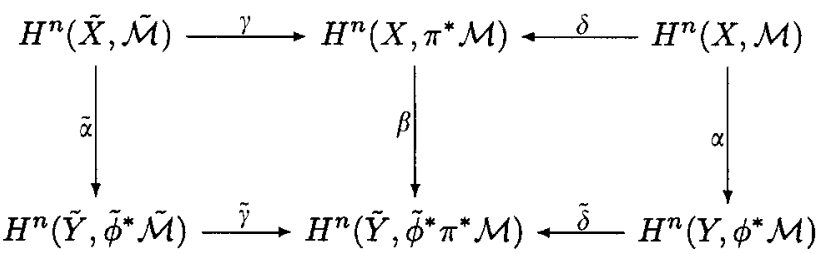

The cokernel of the inclusion $\tilde{\mathcal{M}} \subseteq \pi^{*} \mathcal{M}$ is supported in codimension 1 , so $\gamma$ and $\tilde{\gamma}$ are surjective. The $\beta$ is the zero map, since so is $\tilde{\alpha}$. Now $\delta$ is injective, because $\pi$ is finite and $X$ is normal and $\tilde{\delta}$ is injective by (6.3.2). Therefore $\alpha$ is the zero map.

If $\phi$ is birational, then $\alpha$ is also surjective by (6.3.1), so (6.4.1) follows.

If $X$ has rational singularities, then $\alpha$ is injective by (6.3.2), so (6.4.2) follows.

\section{Arakelov-Parshin boundedness}

DEFINITION 7.1. A morphism, $h: Y \rightarrow C$ is called isotrivial if all but finitely many fibers of $h$ are isomorphic to a fixed variety. Similarly, $h$ is called birationally isotrivial if all but finitely many fibers of $h$ are birational to a fixed variety.

DEFINITION 7.2 [10]. Let $F$ be a normal Gorenstein variety with rational singularities, $\mathcal{L}$ a line bundle on $F$ and $\Gamma$ an effective divisor such that $\mathcal{L}=\mathcal{O}_{F}(\Gamma)$. Let

(1) $\mathcal{C}(\Gamma, N)=\operatorname{coker}\left(\tau_{*} \omega_{\tilde{F}}(-[\tilde{\Gamma} / N]) \rightarrow \omega_{F}\right)$ where $\tau: \tilde{F} \rightarrow F$ is a resolution of singularities such that $\tilde{\Gamma}=\tau^{*} \Gamma$ is a normal crossing divisor.

(2) $e(\Gamma)=\min \left\{N \in \mathbb{N}_{+} \mid \mathcal{C}(\Gamma, N)=0\right\}$

(3) $e(\mathcal{L})=\sup \left\{e(\Gamma) \mid \exists \lambda \in H^{0}(F, \mathcal{L})\right.$ such that $\left.\Gamma=(\lambda=0)\right\}$ 
$e(\mathcal{L})$ will be called the Esnault-Viehweg threshold of $\mathcal{L}$. For properties of $e(\mathcal{L})$ the reader should consult $[41, \S 5.3-4]$

ASSUMPTION 7.3. Let $C$ be a smooth projective curve of genus $g, \Delta \subseteq C$ a finite set of points, regarded as a (reduced) divisor. Let $\delta=\# \Delta$, the number of points in $\Delta$. Let $X$ be an irreducible projective variety of dimension $n$ with rational Gorenstein singularities and $f: X \rightarrow C$ a morphism. Let $D=f^{*} \Delta$.

Assume that $X_{t}$ has rational Gorenstein singularities for $t \in C \backslash \Delta$ and that there exists a simultaneous resolution of $X \backslash D \rightarrow C \backslash \Delta$, i.e., there exists a smooth projective variety $Y$ and a birational morphism $\phi: Y \rightarrow X$, such that $Y \backslash \phi^{*} D \rightarrow C \backslash \Delta$ is smooth. Let $h=f \circ \phi, B=\phi^{*} D$ and let $Y_{\text {gen }}$ denote the general fiber of h. By blowing up $Y$ along a subvariety of $B$ one may assume that $B$ is a (not necessarily reduced) normal crossing divisor.

$r(m)$ will denote the rank of $f_{*} \omega_{X / C}^{m}$. This is equal to the $m$-th plurigenus of the general fiber of $f, P_{m}\left(X_{\text {gen }}\right)$. e $(m)$ will denote the Esnault-Viehweg threshold of $\omega_{X_{\mathrm{gen}}}^{m}$. If $\omega_{X_{\mathrm{gen}}}$ is ample, then $e(m) \leqslant m^{n} K_{X_{\mathrm{gen}}}^{n}+1$ for $m \gg 0$ by [41, 5.12].

Remark 7.3.1. $X_{t}$ has only rational singularities for $t \in C \backslash \Delta$, so the same holds for $X \backslash D$. It is conjectured that a variety with only rational singularities admits a compactification with only rational singularities. Furthermore, if that conjecture holds then the Gorenstein assumption could be avoided as well with a little care. Hence, the assumption on the singularities of $X$ is conjecturally superfluous.

The following lemma gives an effective measure of the positivity of $f_{*} \omega_{X / C}^{m}$. The proof follows parts of the proof of [5, 3.1] very closely, however both the situation and the statement are different from theirs, so the actual proof is included.

PROPOSITION 7.4. Assume that $f$ is nonisotrivial and that $\omega_{X_{\mathrm{gen}}}$ is ample.

Then $\left(f_{*} \omega_{X / C}^{m}\right)^{\otimes e(m) r(m)} \otimes \operatorname{det}\left(f_{*} \omega_{X / C}^{m}\right)^{-1}$ is semi-positive for all $m>0$.

Proof. By [41, 2.8], one may replace $C$ by a finite cover, unramified along $\Delta$, and $X$ by the pull-back family in order to assume that $\operatorname{det} f_{*} \omega_{X / C}^{m}=\mathcal{D}^{e(m)}$ for some invertible sheaf $\mathcal{D}$.

Let $r=r(m)$ and $\pi: Z \rightarrow X^{r}=X \times_{C} X \times_{C} \cdots \times \times_{C} X$ a resolution of singularities. Further let $\rho=f^{r} \circ \pi$. Then $\mathcal{M}=\pi^{*} \omega_{X^{r} / C}=\pi^{*}\left(\otimes \operatorname{pr}_{i}^{*} \omega_{X / C}\right)$ is big by [40, Theorem II].

$f^{r}$ is a Gorenstein morphism and the general fibre has rational singularities, so there are natural injective maps:

$$
\begin{aligned}
& \left.\rho_{*}\left(\mathcal{M}^{m-1} \otimes \omega_{Z / C}\right)\right) \hookrightarrow f_{*}^{r} \omega_{X^{r} / C}^{m}, \\
& f_{*}^{r} \omega_{X^{r} / C}^{m} \hookrightarrow \rho_{*} \mathcal{M}^{m}, \\
& \mathcal{D}^{e(m)} \hookrightarrow\left(f_{*} \omega_{X / C}^{m}\right)^{\otimes r} \simeq f_{*}^{r} \omega_{X^{r} / C}^{m},
\end{aligned}
$$


where (7.4.1) and (7.4.2) are isomorphisms near the generic point of $C$. The composition of (7.4.2) and (7.4.3) gives a section $\sigma \in H^{0}\left(Z, \mathcal{M}^{m} \otimes \rho^{*} \mathcal{D}^{-e(m)}\right)$. Let $A=(a=0)$. Since $\pi$ was an arbirary resolution of singularities one may replace it by further blow-ups, so in particular one may assume that $A$ is a normal crossing divisor.

Let $\mathcal{J} \subseteq \mathcal{O}_{Z}$ be the ideal sheaf defined as

$$
\operatorname{im}\left[\rho^{*} f_{*}^{r} \omega_{X^{r} / C}^{m} \rightarrow \mathcal{M}^{m}\right]=\mathcal{M}^{m} \otimes \mathcal{J} .
$$

Note that the support of $\mathcal{O}_{X} / \mathcal{J}$ is contained in finitely many fibers. By blowing up $\mathcal{J}$ one can assume that it is a line bundle and it is trivial near the general fibre of $\rho$. By [19] $f_{*} \omega_{X / C}^{m} \simeq h_{*} \omega_{Y / C}^{m}$ is semi-positive, hence so is $\mathcal{M}^{m} \otimes \mathcal{J}$, i.e., it is a nef line bundle. Let $\mathcal{K}=\mathcal{M}^{m-1} \otimes \rho^{*} \mathcal{D}^{-1}$. Then

$$
\mathcal{K}^{e(m) m}(-m A)=\mathcal{M}^{e(m) m(m-1)-m^{2}} \supseteq\left(\mathcal{M}^{m} \otimes \mathcal{J}\right)^{e(m)(m-1)-m},
$$

where the inclusion is an equality near the general fiber of $\rho$. Hence, $\mathcal{K}^{e(m)}(-A)$ is nef near the general fiber of $\rho$ and then $\rho_{*}\left(\mathcal{K} \otimes \omega_{Z / C}(-[A / e(m)])\right)$ is semi-positive by $[10$, 1.7]. By [41, 5.14, 5.21],

$$
\mathcal{F}:=\rho_{*}\left(\mathcal{K} \otimes \omega_{Z / C}\left(-\left[\frac{A}{e(m)}\right]\right)\right) \hookrightarrow \rho_{*}\left(\mathcal{K} \otimes \omega_{Z / C}\right)
$$

is an isomorphism near the generic point of $C$. On the other hand, by (7.4.1),

$$
\left.\rho_{*}\left(\mathcal{K} \otimes \omega_{Z / C}\right)\right) \simeq \rho_{*}\left(\mathcal{M}^{m-1} \otimes \omega_{Z / C}\right) \otimes \mathcal{D}^{-1} \hookrightarrow\left(f_{*} \omega_{X / C}^{m}\right)^{\otimes r} \otimes \mathcal{D}^{-1},
$$

is also an isomorphism near the generic point of $C$.

Thus $\mathcal{F} \subseteq\left(f_{*} \omega_{X / C}^{m}\right)^{\otimes r} \otimes \mathcal{D}^{-1}$ which is an equality on an open dense subset of $C$. $\mathcal{F}$ is semi-positive and then so is $\left(f_{*} \omega_{X / C}^{m}\right)^{\otimes r} \otimes \mathcal{D}^{-1}$. Taking the $e(m)$ th power gives the statement.

COROLLARY 7.5. Assume that $h$ is not birationally isotrivial and that $\omega_{y_{\mathrm{gen}}}$ is nef and big. Then $\left(h_{*} \omega_{X / C}^{m}\right)^{\otimes e(m) r(m)} \otimes \operatorname{det}\left(h_{*} \omega_{Y / C}^{m}\right)^{-1}$ is semi-positive for all $m>0$.

LEMMA 7.6. Let $\mathcal{M}$ be a line bundle on $X$ and $\mathcal{N}$ a line bundle on $C$. Assume that $f_{*} \mathcal{M} \otimes \mathcal{N}$ is ample, $\mathcal{M}_{t}=\left.\mathcal{M}\right|_{X_{t}}$ is generated by global sections for $t \in C \backslash \Delta$, and $h^{0}\left(\mathcal{M}_{t}\right)$ is constant. Then

(7.6.1) $\mathcal{M} \otimes f^{*} \mathcal{N}$ is semi-ample with respect to $X \backslash D$.

(7.6.2) If $\mathcal{M}_{t}$ is ample for $t \in C \backslash \Delta$, then $\mathcal{M} \otimes f^{*} \mathcal{N}$ is ample with respect to $X \backslash D$.

Proof. Let $t, s \in C \backslash \Delta$. For $l \gg 0, H^{1}\left(C, \operatorname{Sym}^{l}\left(f_{*} \mathcal{M}\right) \otimes \mathcal{N}^{l} \otimes \mathcal{O}_{C}(-t-s)\right)=0$. Hence, the map $H^{0}\left(C, \operatorname{Sym}^{l}\left(f_{*} \mathcal{M}\right) \otimes \mathcal{N}^{l}\right) \rightarrow \operatorname{Sym}^{l}\left(f_{*} \mathcal{M}\right) \otimes(k(t) \oplus k(s))$ is surjective. 
Since $\mathcal{M}_{t}$ is generated by global sections for $t \in C \backslash \Delta, f^{*} \operatorname{Sym}^{l}\left(f_{*} \mathcal{M}\right) \rightarrow \mathcal{M}^{l}$ is surjective on $X \backslash D$. In particular $f^{*} \operatorname{Sym}^{l}\left(f_{*} \mathcal{M}\right) \otimes\left(\mathcal{O}_{X_{t}} \oplus \mathcal{O}_{X_{s}}\right) \rightarrow \mathcal{M}_{t}^{l} \oplus \mathcal{M}_{s}^{l}$ is surjective.

Now one has the following commutative diagram:

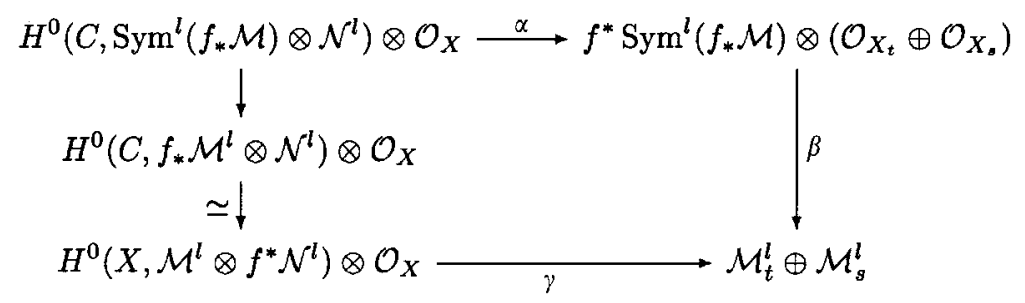

Since $\alpha$ and $\beta$ are surjective, so is $\gamma$. This shows both statements.

DEFINITION 7.7. Let $m_{0}(k)$ be the smallest positive constant such that for all projective varieties, $F$, of dimension $k$ with at most rational singularities and $\omega_{F}$ ample, $\omega_{F}^{m}$ is generated by global sections if $m \geqslant m_{0}(k)$.

Remark 7.7.1. It is known that $m_{0}(k)$ is finite. In fact $m_{0}(k) \leqslant\left(\begin{array}{c}k+1 \\ 2\end{array}\right)+2$ by $[25,5.8]$ (cf. [1]) and Fujita's conjecture predicts that $m_{0}(k) \leqslant k+2$.

THEOREM 7.8. Let $C$ be a smooth projective curve of genus $g, \Delta \subset C$ a finite set of points. Let $f: X \rightarrow C$ be a nonisotrivial morphism satisfying the assumptions made in (7.3) and such that for $t \in C \backslash \Delta, \omega_{X_{t}}$ is ample. Then for all $m \geqslant m_{0}(\operatorname{dim} X-1)$,

$$
\operatorname{deg}\left(f_{*} \omega_{X / C}^{m}\right)=\operatorname{deg}\left(h_{*} \omega_{X / C}^{m}\right) \leqslant m \cdot e(m) \cdot r(m) \cdot[(4 g+\delta-1) \cdot \operatorname{dim} X+2-2 g] .
$$

Proof. $X$ has rational singularities, so $\phi_{*} \omega_{Y / C} \simeq \omega_{X / C}$, and then $h_{*} \omega_{Y / C}^{m} \simeq f_{*} \omega_{X / C}^{m}$.

7.8.1. Let $m \geqslant m_{0}(\operatorname{dim} X-1)$ and $\mathcal{N}$ a line bundle on $C$ such that

$$
\operatorname{deg} \mathcal{N}^{-m e(m) r(m)}<\operatorname{deg}\left(f_{*} \omega_{X / C}^{m}\right) .
$$

Then by (7.4) $f_{*} \omega_{X / C}^{m} \otimes \mathcal{N}^{m}$ is ample on $C . h^{0}\left(X_{t}, \omega_{X_{t}}^{m}\right)$ is constant for $t \in C \backslash \Delta$ by Kawamata-Viehweg vanishing, so $\omega_{X / C} \otimes f^{*} \mathcal{N}$ is ample with respect to $X \backslash D$ by (7.6).

7.8.2. Choose an $l>0$ such that $\omega_{X / C}^{l} \otimes f^{*} \mathcal{N}^{l}$ is generated by global section on $X \backslash D$. By blowing up the base locus of $\omega_{X / C}^{l} \otimes f^{*} \mathcal{N}^{l}$ (contained in $D$ ), one may assume that there exits an effective Cartier divisor $\Gamma$, supported on supp $D$ such that $\omega_{X / C}^{l} \otimes f^{*} \mathcal{N}^{l}(-\Gamma)$ is generated by global sections on the entire $X$.

7.8.3. Let $P \in C \backslash \Delta$. We may assume that $l \geqslant 2 g+\delta$. The linear system $|(2 g+\delta) P-\Delta|$ is base point free, so one can find a reduced effective divisor, 
$\Delta^{\prime} \in|(2 g+\delta) P-\Delta|$ such that $\Delta \cap \Delta^{\prime}=\emptyset$. Let $D=\sum d_{i} D_{i}$ and $l^{\prime}=\underset{i}{\operatorname{lcm}}\left(d_{i}\right) \cdot l$. Let $\Delta^{\prime \prime}=\Delta+\Delta^{\prime}+\left(l^{\prime}-(2 g+\delta)\right) P \in\left|l^{\prime} P\right|$.

Let $\sigma: \tilde{C} \rightarrow C$ be the finite cover obtained by taking the $l^{\prime}$-th root of $\Delta^{\prime \prime}$. Take the fiber product of $\sigma$ with $f$ and $h$. Let $\tilde{X}$ be the normalization of $\tilde{C} \times_{C} X$, $\tilde{f}: \tilde{X} \rightarrow \tilde{C}$, and $\tilde{Y} \rightarrow \tilde{C} \times_{C} Y$ a resolution of singularities such that it is an isomorphism over $\tilde{C} \backslash \sigma^{*} \Delta^{\prime}$.

Note that $\Delta+\Delta^{\prime}$ is a nonempty reduced divisor, so both $\tilde{C}$ and $\tilde{X}$ are irreducible. Let $\quad \bar{\Delta}=\left(\Delta^{\prime}\right)_{\text {red }}, \quad \tilde{\Delta}=\left(\sigma^{*} \bar{\Delta}\right)_{\text {red }}, \quad \tilde{D}=\tilde{f}^{*} \tilde{\Delta} \quad$ and $\quad \tilde{D}_{j}=\left(\pi^{*} D_{j}\right)_{\text {red }}$. Then $\tilde{Y} \backslash \tilde{\phi}^{*} \tilde{D} \rightarrow \tilde{C} \backslash \tilde{\Delta}$ is smooth, $\omega_{\tilde{C}}(\tilde{\Delta}) \simeq \sigma^{*} \omega_{C}(\bar{\Delta})$ and $\bar{\delta}=\# \bar{\Delta}=2 g+\delta+1$. By [26, (2.4), (2.17)] $\delta>0$ if $g=0$, so $2 g-2+\bar{\delta} \geqslant 0$, i.e., $\omega_{\tilde{C}}(\tilde{\Delta}) \simeq \sigma^{*} \omega_{C}(\bar{\Delta})$ is nef. Furthermore, over the smooth locus of $X, \pi^{*} D_{j}=\left(l^{\prime} / \operatorname{gcd}\left(l, d_{j}\right)\right) \tilde{D}_{j}$, so by the definition of $l^{\prime}$, the coefficient of $\tilde{D}_{j}$ is divisible by $l$, hence, s a divisor $\tilde{\Gamma}$ on $\tilde{X}$, supported on $\tilde{D}$, such that $\pi^{*} \Gamma=l \tilde{\Gamma}$. As before, by blowing up the ideal sheaf of $\tilde{\Gamma}$ one may assume that it is a Cartier divisor. Then

$$
\pi^{*}\left(\omega_{X / C}^{l} \otimes f^{*} \mathcal{N}^{l}(-\Gamma)\right)=\pi^{*}\left(\omega_{X / C} \otimes f^{*} \mathcal{N}\right)^{l}(-l \tilde{\Gamma})=\left(\pi^{*}\left(\omega_{X / C} \otimes f^{*} \mathcal{N}\right)(-\tilde{\Gamma})\right)^{l},
$$

so $\pi^{*}\left(\omega_{X / C} \otimes f^{*} \mathcal{N}\right)(-\tilde{\Gamma})$ is semi-ample on $\tilde{X}$ and ample with respect to $\tilde{X} \backslash \tilde{D}$. Finally let $\tilde{\omega}=\pi^{*} \omega_{X / C}(-\tilde{\Gamma})$ and $\tilde{\mathcal{N}}=\sigma^{*} \mathcal{N}$. Using this notation $\tilde{\omega} \otimes \tilde{f}^{*} \tilde{\mathcal{N}}$ is semi-ample on $\tilde{X}$ and ample with respect to $\tilde{X} \backslash \tilde{D}$.

7.8.4. Let $\mathcal{K}=\omega_{C}(\bar{\Delta})^{n}$ where $n=\operatorname{dim} X$ and let $\tilde{\mathcal{K}}=\sigma^{*} \mathcal{K}$. Then by construction

$$
\tilde{\omega} \otimes \tilde{f}^{*}(\tilde{\mathcal{N}} \otimes \tilde{\mathcal{K}}) \subseteq \pi^{*}\left(\omega_{X / C} \otimes f^{*}(\mathcal{N} \otimes \mathcal{K})\right) .
$$

Let $\mathcal{L}=\omega_{X / C} \otimes f^{*}(\mathcal{N} \otimes \mathcal{K}) \otimes f^{*} \omega_{C}(\bar{\Delta})^{-1}$ and $\tilde{\mathcal{L}}=\tilde{\omega} \otimes \tilde{f}^{*} \tilde{\mathcal{N}} \otimes \tilde{f}^{*} \omega_{\tilde{C}}(\tilde{\Delta})^{n-1} \subseteq \pi^{*} \mathcal{L}$. Since $\omega_{\tilde{C}}(\tilde{\Delta})$ is nef, $\tilde{\mathcal{L}}$ and $\tilde{\mathcal{L}} \otimes \tilde{f}^{*} \omega_{\tilde{C}}(\tilde{\Delta})^{-(n-1)}$ are semi-ample on $\tilde{X}$ and ample with respect to $\tilde{X} \backslash \tilde{D}$. Hence, $H^{n}\left(X, \omega_{X / C} \otimes f^{*}(\mathcal{N} \otimes \mathcal{K})\right)=0$ by (6.4.2).

Finally take $\mathcal{N}=\omega_{C}(\bar{\Delta})^{-n} \otimes \omega_{C}$. Then

$$
\mathcal{N} \otimes \mathcal{K} \simeq \omega_{C}, \quad \text { and } \quad \omega_{X / C} \otimes f^{*}(\mathcal{N} \otimes \mathcal{K}) \simeq \omega_{X} .
$$

Since $H^{n}\left(X, \omega_{X}\right) \neq 0$,

$$
\operatorname{deg}\left(f_{*} \omega_{X / C}^{m}\right) \leqslant \operatorname{deg} \mathcal{N}^{-m e(m) r(m)}=\operatorname{deg}\left(\left(\omega_{C}(\bar{\Delta})^{n} \otimes \omega_{C}^{-1}\right)^{m e(m) r(m)}\right) .
$$

COROLLARY 7.9. Under the assumptions of Theorem 7.8, $2 g-2+\delta>0$.

Proof. Follows from Theorems 7.8 and 0.9 .

COROLLARY 7.10. Under the assumptions of Theorem 7.8, for $m \geqslant(\underset{2}{\operatorname{dim} X})+2$.

$$
\frac{\operatorname{deg}\left(f_{*} \omega_{X / C}^{m}\right)}{\operatorname{rk}\left(f_{*} \omega_{X / C}^{m}\right)}=\frac{\operatorname{deg}\left(h_{*} \omega_{Y / C}^{m}\right)}{\operatorname{rk}\left(h_{*} \omega_{Y / C}^{m}\right)} \leqslant 4 \operatorname{dim} X \cdot(2 g-2+\delta) \cdot m \cdot e(m)
$$


Proof. By (7.9) $2 g-2+\delta>0$, so

$$
\operatorname{deg}\left(\omega_{C}(\bar{\Delta})^{\operatorname{dim} X} \otimes \omega_{C}^{-1}\right)=\operatorname{dim} X(4 g-1+\delta)-(2 g-2) \leqslant 4 \operatorname{dim} X(2 g-2+\delta) .
$$

Furthermore, $m_{0}(\operatorname{dim} X-1) \leqslant\left(\begin{array}{c}\operatorname{dim} X \\ 2\end{array}\right)+2$ by [25, 5.8] (cf. [1]).

Remark 7.10.1. By Fujita's conjecture Corollary 7.10 should hold for $m \geqslant \operatorname{dim} X+1$.

COROLLARY 7.11. Let $h: Y \rightarrow C$ be a nonbirationallyisotrivial family such that $Y$ is a smooth projective variety of dimension $n, C$ is a smooth projective curve of genus $g$ and there exists a finite subset $\Delta \subset C$ such that $h$ is smooth over $C \backslash \Delta$. Assume that $\omega_{Y / C}$ is h-nef and h-big. Then

$$
\frac{\operatorname{deg}\left(h_{*} \omega_{Y / C}^{m}\right)}{\operatorname{rk}\left(h_{*} \omega_{Y / C}^{m}\right)} \leqslant 4 \cdot \operatorname{dim} X \cdot(2 g-2+\delta) \cdot m \cdot e\left(\omega_{Y \mathrm{gen}}^{m}\right), \quad \text { for } m \geqslant\left(\begin{array}{c}
\operatorname{dim} X \\
2
\end{array}\right)+2 .
$$

Proof. By the relative base point free theorem [20] there exist morphisms $\phi: Y \rightarrow X$ and $f: X \rightarrow C$ that satisfy Assumption 7.3, so the statement follows from Corollary 7.10.

7.12. Let $\mathfrak{D}_{h}$ denote the moduli functor of canonically polarized normal projective varieties with rational Gorenstein singularities and Hilbert polynomial $h(t)$. Let $\mathfrak{D}_{h}^{(m)}$ denote the submoduli functor of varieties with $\omega_{F}^{m}$ very ample, cf. [41, 1.20, 8.18].

$\mathfrak{D}_{h}^{(m)}$ is open and bounded by definition, so by $[41,8.20]$ there exists a coarse quasi-projective moduli scheme $D_{h}^{(m)}$ for $\mathfrak{D}_{h}^{(m)}$. Furthermore, by [24] there exists an integer $p>0$ and a very ample line bundle $\lambda$ on $D_{h}^{(m)}$ such that for any $f: T \rightarrow S \in \mathfrak{D}_{h}^{(m)}(S)$, the pull back of $\lambda$ on $S$ is $\operatorname{det}\left(f_{*} \omega_{T / S}^{m}\right)^{p}$. Note also that by [41, 5.17] $e\left(\omega_{F}^{m}\right)$ is bounded on $D_{h}^{(m)}$.

Let $\bar{D}_{h}^{(m)}$ be the projective closure of $D_{h}^{(m)}$ corresponding to the embedding given by $\lambda$, and $\mathbf{H}=\operatorname{Hom}\left((C, C \backslash \Delta),\left(\bar{D}_{h}^{(m)}, D_{h}^{(m)}\right)\right)$ the scheme parametrizing morphisms $\Psi: C \rightarrow \bar{D}_{h}^{(m)}$ such that $\Psi(C \backslash \Delta) \subset D_{h}^{(m)}$.

THEOREM 7.13. There exists a subscheme of finite type $T \subset \mathbf{H}$ that contains all points $\left[\Psi: C \rightarrow \bar{D}_{h}^{(m)}\right] \in \mathbf{H}$ induced by morphisms $f: X \rightarrow C \in \mathfrak{D}_{h}^{(m)}(C)$ such that $\left.f\right|_{X \backslash f^{* \Delta}}$ admits a simultaneous resolution.

\section{Acknowledgements}

The lion's share of the work related to this article was done while I enjoyed the hospitality of RIMS at Kyoto University. I would like to express my warm thanks to members, visitors and staff of RIMS for making my stay fruitful and memorable. I would like to thank Shigefumi Mori, especially for making my visit possible and 
also for the interest he took in my work. I would also like to thank him, Stefan Helmke, Stefan Kebekus, Yoichi Miyaoka and Noboru Nakayama for helpful remarks and discussions, Osamu Fujino for saving me from some simple but crucial errors, János Kollár for reading the manuscript and suggesting some very useful improvements and last, but not least, the referee for many helpful remarks.

Commutative diagrams were drawn with the help of Paul Taylor's $\mathrm{T}_{\mathrm{E}} \mathrm{X}$ macro package.

\section{References}

1. Angehrn, U. and Siu, Y.-T.: Effective freeness and point separation for adjoint bundles, Invent. Math. 122 (1995), 291-308.

2. Akizuki, Y. and Nakano, S.: Note on Kodaira-Spencer's proof of Lefschetz theorems, Proc. Japan Acad. 30 (1954), 266-272.

3. Arakelov, S.: Families of algebraic curves with fixed degeneracies. Izv. A.N. SSSR 35 (1971), 1269-1293.

4. Beauville, A.: Le nombre minimum de fibres singulières d'une courbe stable sur $\mathbb{P}^{1}$, Astérisque 86 (1981), 97-108.

5. Bedulev, E. and Viehweg, E.: On the Shafarevich conjecture for surfaces of general type over funcion fields, Invent Math. 139 (2000), 603-615.

6. Catanese, F. and Schneider, M.: Polynomial bounds for abelian groups of automorphisms, Compositio Math. 97 (1995), 1-15.

7. Du Bois, Ph.: Complex de De Rham filtré d'une variété singulière, Bull. Soc. Math. France 109 (1981), 41-81.

8. Ein, L.: Multiplier ideals, vanishing theorems and applications, In: Algebraic Geometry (Santa Cruz 1995) Proc. Sympos. Pure Math. 62, Amer. Math. Soc., Providence, 1997, pp. 203-219.

9. Esnault, H. and Viehweg, E.: Logarithmic de Rham complexes and vanishing theorems, Invent. Math. 86 (1986), 161-194.

10. Esnault, H. and Viehweg, E.: Effective bounds for semipositive sheaves and for the height of points on curves over complex function fields, Compositio Math. 76 (1990), 69-85.

11. Esnault, H. and Viehweg, E.: Lectures on Vanishing Theorems, DMV Seminar vol. 20, Birkhäuser, Basel, 1992.

12. Faltings, G.: Arakelov's Theorem for abelian varieties. Invent. Math. 73 (1983), 337-348.

13. Goresky, M. and MacPherson, R.: Intersection homology II, Invent. Math. 71 (1983), 77-129.

14. Grauert, H. and Riemenschneider, O.: Verschwindungssätze für analytische Kohomologiegruppen auf komplexen Räumen, Invent. Math. 11 (1970), 263-292.

15. Guillén, F., Navarro-Aznar, V., Pascual-Gainza, P. and Puerta, F.: Hyperrésolutions cubiques et descente cohomologique, Lecture Notes in Math. 1335, Springer, New York, 1988.

16. Hartshorne, R.: Residues and Duality, Lecture Notes in Math. 20, Springer, New York, 1966.

17. Hartshorne, R.: On the De Rham cohomology of algebraic varieties. Publ. Math. IHES 45 (1975), 5-99.

18. Kawamata, Y.: A generalisation of Kodaira-Ramanujam's vanishing theorem, Math. Ann. 261 (1982), 43-46. 
19. Kawamata, Y.: Kodaira dimension of algebraic fiber spaces over curves. Invent. Math. 66 (1982), 57-71.

20. Kawamata, Y., Matsuda, K. and Matsuki, K.: Introduction to the minimal model problem, In: T. Oda (ed.), Algebraic Geometry, Sendai, Adv. Stud. Pure Math. 10. Kinokuniya - North-Holland, 1987, pp. 283-360.

21. Kodaira, K.: On a differential geometric method in the theory of analytic stacks, Proc. Nat. Acad. USA 39 (1953), 1268-1273.

22. Kollár, J.: Vanishing theorems for cohomology groups, In: Algebraic Geometry (Bowdoin 1985), Proc. Sympos. Pure Math. 46, Amer. Math. Soc., Providence, 1987, pp. 233-243.

23. Kollár, J.: Subadditivity of the Kodaira dimension: Fibers of general type, In: Algebraic Geometry Sendai 85, Adv. Studies Pure Math. 10, Kinokuniya-North-Holland, 1987, pp. 361-398.

24. Kollár, J.: Projectivity of complete moduli, J. Differential Geom. 32 (1990), 235-268.

25. Kollár, J.: Singularities of pairs, Algebraic Geometry (Santa Curz 1995), Proc. Sympos Pure Math. 62, Amer. Math. Soc., Providence, 1997, pp. 221-287.

26. Kovács, S. J.: Smooth families over rational and elliptic curves, J. Algebraic Geom. 5 (1996), 369-385.

27. Kovács, S. J.: On the minimal number of singular fibres in a family of surfaces of general type, J. Reine Angew. Math. 487 (1997), 171-177.

28. Kovács, S. J.: Algebraic hyperbolicity of fine moduli spaces, J. Algebraic Geom. 9 (2000), $165-174$.

29. Kovács, S. J.: A characterization of rational singularities, Duke Math. J. 102 (2000), 187-191.

30. Kovács, S. J.: Rational, log canonical, Du Bois singularities II: Kodaira vanishing and small deformations, Compositio Math. 121 (2000), 297-304.

31. Migliorini, L.: A smooth family of minimal surfaces of general type over a curve of genus at most one is trivial, J. Algebraic Geom. 4 (1995), 353-361.

32. Navarro-Aznar, V.: Théorèmes d'annulation, Hyperrésolutions cubiques et descente cohomologique, Lecture Notes in Math. 1335, Springer, New York, 1988, pp. 133-160.

33. Oguiso, K. and Viehweg, E.: On the isotriviality of families of elliptic surfaces, $J$. Algebraic Geom. (to appear).

34. Parshin, A.: Algebraic curves over function fields, Izv. A.N. SSSR 32 (1968), 1145-1170.

35. Ramanujam, C. P.: Remarks on the Kodaira vanishing theorem, J. Indian Math. Soc. 36 (1972), 41-51.

36. Shokurov, V. V.: Letters of a bi-rationalist I. A projectivity criterion. In: Y. Kawamata and V. Shokurov (eds) Birational Algebraic Geometry, Contemp. Math. 207, Amer. Math. Soc., Providence, 1997, pp. 143-152.

37. Smith, K. E.: Vanishing, singularities and effective bounds via prime characteristic local algebra, Algebraic Geometry (Santa Cruz 1995), Proc. Sympos. Pure Math. 62, Amer. Math. Soc., Providence, 1997, pp. 289-325.

38. Steenbrink, J. H. M.: Vanishing theorems on singular spaces, Astéristique 130 (1985), 330-341.

39. Viehweg, E.: Vanishing theorems, J.f.r.u.a.Math. 335 (1982), 1-8.

40. Viehweg, E.: Weak positivity and the additivity of the Kodaira dimension II., In: Classification of Algebraic and Analytic Manifolds, Birkhäuser, Basel, 1983, pp. 567-590.

41. Viehweg, E.: Quasi-Projective Moduli of Polarized Manifolds, Springer, New York, 1995.

42. Viehweg, E. and Zuo, K.: On the isotriviality of families of projective manifolds over curves, J. Algebraic Geom. (to appear). 